\title{
REES ALGEBRAS OF IDEALS HAVING SMALL ANALYTIC DEVIATION
}

\author{
SAM HUCKABA AND CRAIG HUNEKE
}

\begin{abstract}
In this article we identify two large families of ideals of a CohenMacaulay (sometimes Gorenstein) local ring whose Rees algebras are CohenMacaulay. Our main results imply, for example, that if $(R, M)$ is a regular local ring and $P$ is a prime ideal of $R$ such that $P^{n}$ is unmixed for all $n \geq 1$, then the Rees algebra $R[P t]$ is Cohen-Macaulay if either $\operatorname{dim}(R / P)=2$, or $\operatorname{dim}(R / P)=3, R / P$ is Cohen-Macaulay, and $R / P$ is integrally closed.
\end{abstract}

\section{INTRODUCTION}

Over the past 10-15 years, several graded rings associated with a local ring $R$ and ideal $I$ have been intensively studied. These graded rings include the associated graded ring $\operatorname{gr}(I, R)=\bigoplus_{n \geq 0} I^{n} / I^{n+1}$, the Rees algebra $R[I t]$ (the subring of the polynomial ring $R[t]$ given by $\left.\bigoplus_{n>0} I^{n} t^{n}\right)$, the extended Rees algebra $R\left[I t, t^{-1}\right]$, the symbolic "blowup" $\bigoplus_{n \geq 0} I^{(n)} t^{n}$, and approximations to these rings, most notably the symmetric algebra of $I$ and $I / I^{2}$. All of these algebras encode a great deal of information about the ideal $I$ and how it sits in $R$.

Of all these rings, we feel that the Rees algebra $R[I t]$ has the most central role, although originally more attention was focused on the associated graded ring and the extended Rees algebra. For instance, when both the Rees algebra and $R$ are Cohen-Macaulay (C-M) so is the associated graded ring (cf. [Hun1, Proposition 2.2]) and the extended Rees algebra. Furthermore the determination of whether the powers of $I$ are unmixed reduces to a calculation of analytic spreads (see (1.2) below). It is easy to see from the isomorphism of $R\left[I t, t^{-1}\right] /\left(t^{-1}\right)$ with $\operatorname{gr}(I, R)$ that the extended Rees algebra is C-M iff the associated graded ring is C-M. However, the statement that the Rees algebra is $\mathrm{C}-\mathrm{M}$ packs more information and is logically stronger than the assertion that the graded ring is C-M. Moreover, it is convenient to study the Rees algebra (as opposed to the associated graded ring or the extended Rees algebra) for other reasons. The presentation of the Rees algebra is often much simpler than that of the other two algebras. Studying the extended Rees algebra can also be burdensome since on the face of it one must worry about negative degrees in the obvious grading. In addition $X=\operatorname{Proj}(R[I t])$ is the blowup of $I$ and plays

Received by the editors November 5, 1990 and, in revised form, June 30, 1991.

1991 Mathematics Subject Classification. Primary 13H10; Secondary 13C05, $13 \mathrm{C} 13$.

The second author was partially supported by the National Science Foundation. 
an extremely important role in the birational study of algebraic varieties. The Cohen-Macaulay property of the Rees algebra can be translated into a vanishing statement about the global cohomology of $X$. Such vanishing results are quite useful, both in algebraic geometry and commutative algebra.

Our main interest in this paper is in proving certain special classes of ideals, those of small analytic deviation, have Cohen-Macaulay Rees algebras. Our results extend those of a number of papers, e.g., [Br2, C-N, E-H, GS2, Hun 1, 2, $\mathrm{HH}$, and SV] among others. Before introducing some notation to explain our main results, we make some comments about the study of Rees algebras.

Rees algebras were originally introduced by David Rees to study several problems in commutative algebra. In [R1], Rees used the (extended) symbolic blowup algebra of a height one prime to give a counterexample to Zariski's proposed generalization of Hilbert's 14th problem. In [R2 and R3], Rees used the extended Rees algebra to give proofs of two classic theorems in commutative algebra; the Artin-Rees lemma, and his famous criterion for a local ring to be analytically unramified. One of the underlying themes in the study of the Rees algebra of an ideal, besides the obvious fact that Rees algebras provide a wealth of interesting examples, was set by Rees in his early work. Namely, that Rees algebras of ideals in noetherian rings capture in another noetherian ring a great deal of information about how the ideal sits in the ring and how its powers change. Ever since the introduction of the Hilbert function and the idea of multiplicity, it has been recognized that understanding how ideals grow and at what rate they grow as we raise them to powers is of fundamental importance in commutative noetherian ring theory. When the Rees algebra is Cohen-Macaulay we can capture this information about the growth of $I$ in a very precise and tightly controlled manner. In particular, when the Rees algebra is Cohen-Macaulay we can study, in a much more precise way than in general, the behavior of the powers of the ideal as we specialize the base ring by factoring out other ideals (cf. [EH, Corollary 1.6]). One example of a type of information which we obtain once we know the Rees algebra of an ideal $I$ in a local noetherian ring $R$ is Cohen-Macaulay is a criterion for deciding whether the powers of $I$ are unmixed. As we will use some of this material repeatedly in this paper, we recall some of the basic concepts.

If $(R, M)$ is a noetherian local ring and $I$ is an ideal of $R$, then the analytic spread of $I$ is defined to be $\operatorname{dim}\left(\bigoplus_{n \geq 0} I^{n} / M I^{n}\right)$, where $\operatorname{dim}()$ denotes the Krull dimension (cf. [NR]). Let $l(I)$ denote the analytic spread of $I$, and $\mathrm{ht}(I)$ denote the height of $I$. In general, one has the inequalities $h t(I) \leq$ $l(I) \leq \operatorname{dim}(R)$. A fundamental result of Burch [Bu] which we will often make use of, relates the depth of the powers of $I$ to the analytic spread of $I$. Namely, she showed the inequality

$$
l(I) \leq \operatorname{dim}(R)-\inf _{n}\left\{\operatorname{depth}\left(R / I^{n}\right)\right\} .
$$

It is of interest to know when this inequality is an equality, as in general the analytic spread of an ideal is much easier to compute than the depth of powers of an ideal. When the Rees algebra $R[I t]$ is Cohen-Macaulay, this inequality becomes an equality [EH, Proposition 3.3]. One nice use of $(1.1)$ is the following necessary condition for the powers of a prime ideal $I$ to be unmixed (recall that an ideal $I$ is said to be unmixed if for all associated primes $Q$ of $I$, 
$\operatorname{dim}(R / Q)=\operatorname{dim}(R / I)):$ for all primes $P$ such that $P$ properly contains $I$,

$$
l\left(I_{P}\right)<\operatorname{dim}\left(R_{P}\right) .
$$

This condition is also sufficient when one has equality locally for all primes $P$ containing $I$ in (1.1). In particular whenever the Rees algebra is C-M, (1.2) is a necessary and sufficient condition for the powers of $I$ to be unmixed. When $I$ is a prime, to say its powers are unmixed is the same as saying that $I^{n}=I^{(n)}$ for all $n \geq 1$, where $I^{(n)}$ is the $n$th symbolic power of $I$, which by definition is the $I$-primary component of $I^{n}$.

Cowsik and Nori [CN] made use of Burch's inequality (1.1) to prove the following theorem, which we shall later use.

Theorem 1.3. Let $(R, M)$ be a Cohen-Macaulay local ring, and let $P$ be a prime ideal in $R$ such that $R_{P}$ is regular. Then $P$ is generated by a regular sequence if and only if $l(P)=\mathrm{ht}(P)$. Consequently, if $R / P^{n}$ is $C-M$ for all $n \geq 1$, then $P$ is generated by a regular sequence.

For instance, if $R$ is a regular local ring and $P$ is a prime ideal with $\operatorname{dim}(R / P)=1$, this theorem implies that every power of $P$ is unmixed iff $P$ is generated by a regular sequence. For regular local rings of dimension three, this statement can be improved to the following: if any power greater than one of $P$ is unmixed, then $P$ is generated by a regular sequence (in which case all powers of $P$ are unmixed); cf. [Hun3, 2.5].

A basic concept which we will use is that of the analytic deviation of an ideal. In $[\mathrm{HH}]$ we defined the analytic deviation of $I$, denoted $\operatorname{ad}(I)$, to be the difference between the analytic spread of $I$ and the height of $I$. Symbolically, $\operatorname{ad}(I)=l(I)-\mathrm{ht}(I)$. The paper [HH] began the study of ideals of analytic deviation 1 or 2 , which we will continue in this paper. If $\operatorname{ad}(I)=0, I$ is said to be equimultiple. Such ideals include all $M$-primary ideals in a noetherian local ring $(R, M)$, but also include many other ideals. These ideals have been intensively studied, partly because they arise in Hironaka's proof of resolution of singularities. Hironaka's work gave even more impetus to the study of Rees algebras and graded rings by developing his ideas of normal flatness and standard basis. We refer the reader to the book of Hermann, Ikeda, and Orbanz [HIO] for a wealth of information on this topic and for other references. In particular Chapter V of that book studies the Cohen-Macaulay property of the Rees algebra of equimultiple ideals.

In this paper, however, our roots do not lie in the study of equimultiplicity, but rather lie in the work of many people, especially Herzog, Simis, and Vasconcelos [HSV1, 2], concerning the Rees algebra of an ideal of small deviation, i.e., the difference $\mu(I)-h t(I)$ is small, where $\mu(I)$ represents the minimal number of generators of $I$. This difference in the starting point is easily explained. Most of our theorems require that the base ring $R$ is Cohen-Macaulay. Furthermore, we are mainly interested in ideals which are prime and generically generated by a regular sequence, i.e., in prime ideals $P$ such that $R_{P}$ is regular. For such ideals if $P$ is equimultiple (that is has analytic deviation 0 ) then by Theorem 1.3 we find that $P$ is generated by a regular sequence. In this case it is well known that the Rees algebra is Cohen-Macaulay, and the powers of such an ideal are completely understood. On the other hand, if the deviation of $I$ is 1 or 2 , then there is a great deal of interesting work concerning the 
powers of $I$, and its associated graded algebras, e.g., see [Br2, GS2, HRZ, Hun3, HSV1, HSV2, AH]. In many ways the most systematic treatment is due to Herzog, Simis, and Vasconcelos who were able to connect the low deviation with a crucial property of the ideal; that of having Cohen-Macaulay Koszul homology, a condition which is now called strongly Cohen-Macaulay (cf. [Hun4]). This also allowed a treatment of the Rees algebras of ideals in the linkage class of a complete intersection. A side profit from this study was the realization that Rees algebras provide a type of generic residual intersection (see [Hun4, HU, and HVV]); as such, understanding when the Rees algebra is Cohen-Macaulay can often provide information about when any residual intersection of the ideal is Cohen-Macaulay. The concept of a residual intersection is a generalization of linkage. We will discuss linkage below. From an opposite side, constructions arising from this point of view provided examples of rings (which are Rees algebras) that are non-Cohen-Macaulay UFDs (see [HH, 4.7] for example). Our basic point of view in this paper is to prove theorems about when the Rees algebra is Cohen-Macaulay. Instead of requiring that the ideal $I$ have few generators, we only require that $I$ has small analytic deviation. Although we require some technical hypotheses, we note two corollaries which follow from our main results and which do not need any more notation to explain.

Corollaries 2.23 and 4.22. Let $(R, M)$ be a regular local ring and let $P$ be a prime in $R$ such that all powers of $P$ are unmixed. Then the Rees algebra $R[P t]$ is $C-M$ in either of the following two cases:

(i) $\operatorname{dim}(R / P)=2$.

(ii) $\operatorname{dim}(R / P)=3, R / P$ is normal, and $R / P$ is $C-M$.

This result is new and somewhat surprising.

Through the development of the field of commutative algebra over the last 30 years, Rees algebras have come to be recognized as objects of interest in and of themselves. In the mid-seventies, it was shown by DeConcini, Eisenbud, and Procesi [DEP] that the extended Rees algebra also provided a framework for creating deformations to the "normal cone" (meaning the associated graded ring) while preserving a "straightening law". At the same time work of Brodmann, Herrmann, Herzog, Goto, Ikeda, Itoh, Katz, McAdam, Morales, Ratliff, Rees, Robbiano, Sally, Simis, Schenzel, Shimoda, Trung, Valla, Vasconcelos, and many others used Rees algebras to study a wide range of topics. The Rees algebra is a convenient package which carries a great deal of information about the ring $R$, the ideal $I$, and how $I$ lies inside $R$. On the other hand, the search for perfect ideals and Cohen-Macaulay rings has occupied many commutative algebraists for the past 25 years or so. This paper hopes to contribute to both of these goals.

One of our main results in case the analytic deviation is one is the following corollary. We say that an ideal $J$ in a local ring is a complete intersection if it is generated by a regular sequence.

Corollary 2.21. Let $R$ be a $C-M$ local ring and $P$ a prime ideal of $R$. Assume that $\operatorname{ad}(P)=1, \operatorname{ht}(P) \geq 1$, and $P_{Q}$ is a complete intersection for every $Q \supseteq P$ with $\operatorname{ht}(Q / P)=1$. Then the following are equivalent.

(i) $\operatorname{depth}(R / P) \geq \operatorname{dim}(R / P)-1$.

(ii) $\operatorname{gr}(P, R)$ is $C-M$. 
(iii) $R[P t]$ is $C-M$.

In [HH, Corollary 2.10], (i) and (ii) were shown to be equivalent. Condition (iii) is what is new in (2.21). For ideals of analytic deviation 2, one of our main results is

Corollary 4.20. Let $(R, M)$ be a Gorenstein local ring and $P$ a prime ideal of $R$ such that $\operatorname{ad}(P)=2$ and $\operatorname{ht}(P) \geq 2$. Assume that $R / P$ is $C-M$, and $P_{Q}$ is a complete intersection for all prime ideals $Q$ such that $\operatorname{ht}(Q / P)=2$. Then $R[P t]$ is $C-M$.

The proof of Corollary 4.20 is considerably more difficult than that of Corollary 2.21. One of the keystones of the proofs of both of these corollaries is that the reduction number of the ideal in both cases is one. We recall the definition of reduction and reduction number.

Definition 1.4 [NR]. An ideal $J \subseteq I$ is said to be a reduction of $I$ if there exists an $n \geq 0$ such that $J I^{n}=I^{n+1}$. A reduction $J$ is said to be a minimal reduction of $I$ if $J$ is minimal (under inclusion) with respect to being a reduction of $I$. If $J$ is a reduction of $I$, then the reduction number of $I$ with respect to $J$ is defined to be

$$
r_{J}(I)=\min \left\{n \geq 0 \mid J I^{n}=I^{n+1}\right\} .
$$

The reduction number of $I$ is defined to be

$$
r(I)=\min \left\{r_{J}(I) \mid J \text { a reduction of } I\right\} \text {. }
$$

One of the surprising results of $[\mathrm{HH}]$ was that ideals $I$ of analytic deviation 1 or 2 often have reduction number 1 . Here is a combined version of Corollaries 2.5 and 3.3 from that paper.

Theorem 1.5. (i) Let $(R, M)$ be a $C-M$ local ring with $R / M$ infinite and $P$ a prime ideal of $R$ such that $\operatorname{ad}(P)=1, \operatorname{ht}(P) \geq 1$, and $P_{Q}$ is a complete intersection for all primes $Q$ with $\mathrm{ht}(Q / P)=1$. Then $r_{J}(P) \leq 1$ for every minimal reduction $J$ of $P$.

(ii) Let $(R, M)$ be a Gorenstein local ring with $R / M$ infinite and let $P$ be a prime ideal of $R$ such that $\operatorname{ad}(P)=2, \operatorname{ht}(P) \geq 2, R / P$ is $C-M$, and $P_{Q}$ is a complete intersection for all primes $Q$ with $\mathrm{ht}(Q / P)=2$. Then $r_{J}(P) \leq 1$ for every minimal reduction $J$ of $P$.

In this paper, we assume that the reduction number is one in the statements of the main theorems; however, applying Theorem 1.5 allows us to obtain the corollaries stated above (which do not require an assumption on the reduction number).

We will study the powers of $I$ by means of analyzing a reduction $J$ of $I$ having a small number of generators. This is possible due to the fact that if $(R, M)$ is local and $R / M$ is infinite, then any minimal reduction $J$ of $I$ has $l(I)$ minimal generators, i.e., $\mu(J)=l(I)$. Hence if $\operatorname{ad}(I)=k$, then the deviation of $J$ is $k$. Of course, replacing $I$ by a minimal reduction $J$ is not without problems; one of the main problems to overcome is that even if we assume $R / I$ is $\mathrm{C}-\mathrm{M}$, it will often happen that $\operatorname{depth} R / J=0$ for a minimal reduction $J$ of $I$. In fact we must choose our reductions very carefully. In 
particular we make use of basic element theory. Our choices for generators of the minimal reductions is the subject of $\S 3$ of this paper.

We should remark that ideals with small analytic deviation can have arbitrarily large deviation, even in regular local rings. For example,

Example 1.6. Let $R=K[U, V, W, Z]_{(U, V, W, Z)}$ where $K$ is a field and $U$, $V, W, Z$ are variables. Let $P$ be a height 2 prime ideal of $R$ that contains the element $f=(U W-V Z)$. If $P$ is not a complete intersection, then $\operatorname{ad}(P)=$ 1 (see [HH, Proposition-Example 4.1]). Such primes can require arbitrarily many generators (see [HH, Remark 4.4(i)]). Special cases of this example have been studied by Schenzel [Sc], and Morales and Simis [MS].

The idea of focusing attention upon the reduction number to study the Rees algebra is not new; e.g., Sally [Sa] studied the associated graded ring of the maximal ideal in cases where the reduction number is small. Goto and Shimoda [GS] obtained results about when $\operatorname{gr}(I, R)$ C-M implies $R[I t]$ C-M in terms of the reduction number of $I$. Huneke and Sally [HS] used the fact that integrally closed ideals in a 2-dimensional regular local ring have reduction number 1 (see [LT, Proposition 5.5]) to prove their Rees algebras were C-M with minimal multiplicity; a result which allowed them to study the associated Rees valuations of these ideals. Verma has studied this problem in more depth [Ve].

A natural question is, why does this paper only study $\operatorname{ad}(I) \leq 2$ ? What about $\operatorname{ad}(I)=3$ or higher? The main reason the results do not go through for ideals of higher analytic deviation, at least in terms of the method of proof, has to do with linkage and residual intersections. As we will use linkage in the proofs both implicitly and explicitly, we take space here to recall the basic facts we shall use (cf. [PS]).

Definition 1.7. Let $(R, M)$ be a C-M local ring and let $I, J$ be two ideals of $R$. We say that $I$ and $J$ are linked if there exists a regular sequence $x_{1}, \ldots, x_{c} \in I \cap J$ such that $\left(\left(x_{1}, \ldots, x_{c}\right): I\right)=J$ and $\left(\left(x_{1}, \ldots, x_{c}\right): J\right)=I$.

We will use the following (cf. [PS, Propositions 2.1 and 1.3]):

Remark 1.8. Let $(R, M)$ be a Gorenstein local ring and let $I$ be an unmixed ideal. Let $x_{1}, \ldots, x_{c}$ be a maximal regular sequence in $I$ and set $J=\left(\left(x_{1}, \ldots, x_{c}\right): I\right)$. Then $I$ and $J$ are linked, and $R / I$ is C-M iff $R / J$ is C-M.

In Remark 1.8 the assumption that $R$ be Gorenstein is necessary, and this requires us to assume $R$ is Gorenstein in $\S 3$.

One of the ingredients in our proofs is understanding

$$
\operatorname{depth} R /\left(\left(x_{1}, \ldots, x_{l}\right): I\right)
$$

where $x_{1}, \ldots, x_{l}$ are part of a minimal reduction of $I$. It turns out that when $l$ is small compared with $\mathrm{ht}(I)$, computing such depths is a tractable problem, but as $l$ grows larger, computing these depths is very difficult without enhancing our assumptions significantly. This difficulty has a lot to do with the fact one must assume the ring $R$ in Remark 1.8 is Gorenstein (as opposed to C-M), and brings one into the study of residual intersections (see [Hun4, HU, HVV]). We recall the definition: If $I$ is an ideal of height $d$ and $J$ is a proper subideal of $I$ minimally generated by $s \geq d$ elements, then the ideal $A=(J: I)$ is called an $s$-residual intersection if $\mathrm{ht}(A) \geq s$. 
We end the introduction by collecting some more of the terminology and results we will be using throughout this paper.

The following remarks will be used frequently, sometimes without explicit reference.

Remark 1.9 (called the "depth lemma" in [EG]). Let $R$ be a noetherian local ring and $0 \rightarrow A \rightarrow B \rightarrow C \rightarrow 0$ an exact sequence of finitely generated $R$ modules. Then either

(a) $\operatorname{depth}(A) \geq \operatorname{depth}(B)=\operatorname{depth}(C)$, or

(b) $\operatorname{depth}(B) \geq \operatorname{depth}(A)=\operatorname{depth}(C)+1$, or

(c) $\operatorname{depth}(C)>\operatorname{depth}(A)=\operatorname{depth}(B)$.

Proof. This follows easily from the characterization of depth in terms of the vanishing of Ext, and the long exact sequence for Ext.

Remark 1.10 (McAdam's Avoidance Lemma, [Mc, Theorem 5]). Let $R$ be a commutative ring such that $R / M$ is infinite for every maximal ideal $M$. Let $J_{1}, \ldots, J_{n}$ be not necessarily distinct ideals of $R$, and let $c_{1}, \ldots, c_{n}$ be elements of $R$. If $I \subseteq J_{1}+c_{1} \cup J_{2}+c_{2} \cup \cdots \cup J_{n}+c_{n}$, then for some $i=1,2, \ldots, n$, $I+R c_{i} \subseteq J_{i}$.

If $I \subseteq R$ and $x \in R$ we will usually let $x^{\prime}$ denote the leading form of $x$ in $\operatorname{gr}(I, R)$. That is, $x^{\prime}$ is the image of $x$ in $I^{n} / I^{n+1}$ where $x \in I^{n}$, $x \notin I^{n+1}$. We will also say an ideal $I$ is generically a complete intersection if $I_{P}$ is generated by a regular sequence of length $\mathrm{ht}(I)$ for all $P \in \min (R / I)=$ \{primes minimal over $I$ \} . Local ring will always mean noetherian local ring.

Obviously it is important for us to understand the existence of regular sequences in both the Rees algebra and the associated graded ring. We will sometimes use a result of Valabrega and Valla [VV, Theorem 2.3]:

Remark 1.11. Let $R$ be a noetherian ring, $I$ an ideal of $R$, and let $x_{1}, \ldots, x_{n}$ be a regular sequence in $R$. Then the leading forms of the $x_{i}$ form a regular sequence in $\operatorname{gr}(I, R)$ iff for all $i=1, \ldots, n$ and all $m \geq 1$,

$$
\left(x_{1}, \ldots, x_{i}\right) \cap I^{m}=\sum_{j=1}^{i} I^{m-p_{j}} x_{j},
$$

where $p_{j}=\operatorname{deg}\left(x_{j}^{\prime}\right)$, the degree in $\operatorname{gr}(I, R)$ of the initial form of $x_{j}$.

We will also need to use a well-known result of Matijevic and Roberts [MR, Theorem] and Hochster and Ratliff [HRa, Proposition 4.10].

Remark 1.12. If $R$ is a graded ring and $R_{P}$ is C-M for every graded prime ideal of $R$ (equivalently if $R_{M}$ is C-M for every graded maximal ideal of $R$ ), then $R$ is C-M.

Acknowledgment. We thank the referee for suggestions and comments concerning the presentation of the material.

\section{AnAlytic Deviation 1}

In this section we will prove a strengthened version of [HH, Theorem 2.9]. Here is the statement of our result. 
Theorem 2.1. Let $(R, M)$ be a $C$-M local ring such that $R / M$ is infinite and let $I$ be an ideal of $R$. Assume that $\mathrm{ht}(I) \geq 1, I$ is generically a complete intersection, $\operatorname{ad}(I)=1$, and $r(I) \leq 1$. Then the following are equivalent:

(i) $\operatorname{depth}(R / I) \geq \operatorname{dim}(R / I)-1$,

(ii) $\operatorname{gr}(I, R)$ is $C-M$,

(iii) $R[I t]$ is $C-M$.

Before proving the theorem we make several remarks and discuss a number of lemmas that will be needed for the proof.

Remark 2.2. If $\operatorname{ad}(I)=0$ then Theorem 2.1 is true also. This follows (after choosing a minimal reduction of $I$ carefully as in the proof below) from Remark 1.11 along with a generalized version of [Gs1, Remark 3.10] (see [HIO, Chapter V]).

Remark 2.3. The assumptions for the stated equivalence in Theorem 2.1 are not necessary, even in the case $\operatorname{ad}(I)=0$ (see [Gs1, Remark 3.10]). It is hard however to move away from a small reduction number condition, especially in the case of positive analytic deviation. Our techniques make heavy use of the condition $r(I) \leq 1$. A relaxation of this condition would appear to require significant changes in technique.

Remark 2.4. The assumption in Theorem 2.1 that $r(I) \leq 1$ is often satisfied as is pointed out in Theorem 1.5(i) of the introduction.

The following lemmas found in $[\mathrm{HH}]$ will be used in the proof.

Lemma 2.5 [HH, Remark 2.1(iii)]. Let $R$ be a $C$-M local ring and I an ideal of $R$. Suppose that $\left\{a_{1}, \ldots, a_{d}\right\}$ is a regular sequence contained in $I$ that generates $I$ generically, and $c \in I$ such that $\sqrt{I}=\sqrt{\left(a_{1}, \ldots, a_{d}, c\right)}$. Then $\left(\left(a_{1}, \ldots, a_{d}\right)^{m}: c^{n}\right) \cap I^{m}=\left(a_{1}, \ldots, a_{d}\right)^{m}$ for all positive integers $m$ and $n$.

Lemma 2.6 [HH, Lemma 2.6]. Let $R$ be a $C$-M local ring and $I$ an ideal of $R$. Suppose that $\mathrm{ht}(I)=d \geq 1$ and $\operatorname{dim}(R / I) \geq 1$. Let $\left\{a_{1}, \ldots, a_{d}\right\}$ be a regular sequence contained in $I$ that generates $I$ generically and let $c \in I$ be a regular element such that $\left(a_{1}, \ldots, a_{d}, c\right) I=I^{2}$. Then,

(i) $\operatorname{depth}\left(R /\left(\left(a_{1}, \ldots, a_{d}\right)^{i}+I^{n}\right)\right) \geq \min \left\{\operatorname{depth}\left(R / I^{i}\right), \operatorname{dim}(R / I)-1\right\}$ for all integers $n \geq 1$ and $i=1, \ldots, n$;

(ii) $\left(a_{1}, \ldots, a_{d}\right)^{i} \cap I^{n}=\left(a_{1}, \ldots, a_{d}\right)^{i} I^{n-i}$ for all integers $n \geq 1$ and $i=$ $1, \ldots, n$.

Remark 2.7. If $i=1$ in Lemma 2.6(ii), then the equality there can be expanded to include the cases $\left(a_{1}, \ldots, a_{j}\right) \cap I^{n}=\left(a_{1}, \ldots, a_{j}\right) I^{n-1}$ for $1 \leq j \leq d$. This follows from [VV, Proposition 2.6]. We will use this observation within some of the proofs below.

The next lemma will play a key role in the inductive step of part of the proof of Theorem 2.1. It will also resurface in $\S 3$ when we consider the analytic deviation 2 case.

Lemma 2.8. Let $(R, M)$ be a $C$-M local ring and $I$ an ideal of $R$. Assume that $\mathrm{ht}(I)=s$ and that $a_{1}, \ldots, a_{s} \in I$ such that $\left\{a_{1}, \ldots, a_{s}\right\}$ is an $R$-regular sequence satisfying $\left(a_{1}, \ldots, a_{s}\right) \cap I^{n}=\left(a_{1}, \ldots, a_{s}\right) I^{n-1}$ for all $n \geq 1$. Assume also that $\operatorname{depth}\left(R /\left(\left(a_{1}, \ldots, a_{s}\right)+I^{n}\right)\right)>0$ for all $n \geq 1$. Then there exists an 
element $x \in M \backslash I$ such that $x^{\prime}$ is a $\operatorname{gr}(I, R)$-regular element. Furthermore if $\mathscr{P}$ is any finite set of prime ideals of $R$ and $\mathscr{P}$ does not contain $M$, then $x$ as above may be chosen so that $x \notin Q$ if $Q \in \mathscr{P}$.

Proof. Set

$$
\mathscr{B}=\left\{Q \in \operatorname{Spec}(R) \mid Q \in \operatorname{Ass}\left(R /\left(\left(a_{1}, \ldots, a_{s}\right)+I^{n}\right)\right) \text { for some } n \geq 1\right\} .
$$

It follows from [ $\mathrm{Br} 1$, Main Result] that $\mathscr{B}$ is a finite set. We also have that $M \notin \mathscr{B}$ because $\operatorname{depth}\left(R /\left(\left(a_{1}, \ldots, a_{s}\right)+I^{n}\right)\right)>0$ for all $n \geq 1$. Using prime avoidance we may choose $x \in M$ such that $x \notin Q$ if $Q \in \mathscr{P} \cup \mathscr{B}$ where $\mathscr{P}$ is a finite set of primes with $M \notin \mathscr{P}$. We claim that $x^{\prime}$ is a $\operatorname{gr}(I, R)$-regular element. The proof of this claim is by induction on $s=\mathrm{ht}(I)$. Let $s=0$. Then by our choice of $x$ we have that $\left(I^{n}: x\right)=I^{n}$ (which is equivalent to $x^{\prime}$ being a $\operatorname{gr}(I, R)$-regular element). Assume that $x^{\prime}$ is a $\operatorname{gr}(I, R)$-regular element if $1 \leq \mathrm{ht}(I)<s$ and $x$ is chosen as above, and suppose that $\mathrm{ht}(I)=s$. By the equality $\left(a_{1}, \ldots, a_{s}\right) \cap I^{n}=\left(a_{1}, \ldots, a_{s}\right) I^{n-1}$ for all $n \geq 1$ we have that $\left\{a_{1}^{\prime}, \ldots, a_{s}^{\prime}\right\}$ is a $\operatorname{gr}(I, R)$-regular sequence by Remark 1.11. In particular $a_{1}^{\prime}$ is a $\operatorname{gr}(I, R)$-regular element, hence $\left(a_{1}\right) \cap I^{n}=a_{1} I^{n-1}$ for all $n \geq 1$. Let $r \in\left(I^{n}: x\right)$. We show by induction on $n$ that $r \in I^{n}$. If $n=1$ then this is clear. Let $n>1$. Set $I_{0}=I /\left(a_{1}\right)$ and let $r_{0}, x_{0}$ denote the images of $r$ and $x$ modulo $\left(a_{1}\right)$. Then $r_{0} \in\left(I_{0}^{n}: x_{0}\right)=I_{0}^{n}$ by the induction hypothesis on $s$. Thus $r \in\left(a_{1}\right)+I^{n}$. Write $r=a_{1} b_{1}+y$ where $b_{1} \in R$ and $y \in I^{n}$. Then $(r-y) x=a_{1} b_{1} x \in\left(a_{1}\right) \cap I^{n}=a_{1} I^{n-1}$, therefore $b_{1} \in\left(I^{n-1}: x\right)=I^{n-1}$ by the inductive hypothesis on $n$. This implies that $r \in I^{n}$, completing the proof of the lemma.

Remark 2.9. The ideas involved in the proof of Lemma 2.8 can be adapted to give the following result:

Under the same assumptions as those given in Lemma 2.8 and assuming also that $\operatorname{depth}\left(R /\left(\left(a_{1}, \ldots, a_{s}\right)+I^{n}\right)\right) \geq m$ for all $n \geq 1$ where $m$ is some fixed positive integer, there exists $x_{1}, \ldots, x_{m} \in M \backslash I$ such that $\left\{a_{1}^{\prime}, \ldots, a_{s}^{\prime}, x_{1}^{\prime}, \ldots, x_{m}^{\prime}\right\}$ is a $\operatorname{gr}(I, R)$-regular sequence.

The idea for the proof of $(2.10)$ is to use induction on $m$ with Lemma 2.5 providing the selection of $x_{1}$ if $m=1$. To finish the case $m=1$ one shows directly that $\left\{a_{1}^{\prime}, \ldots, a_{s}^{\prime}, x_{1}^{\prime}\right\}$ is a $\operatorname{gr}(I, R)$-regular sequence. For the general case one again uses Lemma 2.8 to choose $x_{1}$ then by using that $x_{1}^{\prime}$ is a $\operatorname{gr}(I, R)$-regular element it is possible to work modulo $\left(x_{1}\right)$ and preserve the assumptions. The inductive hypothesis then allows one to choose the other elements, then everything is lifted back to $R$.

We now present another lemma that will be important for some of our induction arguments (both here and in $\S 3$ ).

Lemma 2.11. Let $(R, M)$ be a $C$-M local ring and $I$ an ideal of $R$. Assume that $\mathrm{ht}(I) \geq 1$ and let $a \in I \backslash I^{2}$ such that $a^{\prime}$ is a $\operatorname{gr}(I, R)$-regular element. Set $R_{1}=R /(a)$ and $I_{1}=I /(a)$. If $R_{1}\left[I_{1} t\right]$ is $C-M$, then $R[I t]$ is $C-M$.

Proof. Note first that $a$ is an $R$-regular element because $a^{\prime}$ is a $\operatorname{gr}(I, R)$ regular element. Consider the exact sequence

$$
0 \rightarrow(a, a t) /(a t) \rightarrow R[I t] /(a t) \rightarrow R[I t] /(a, a t) \rightarrow 0
$$


of $R[I t]$-modules. In order to show that $R[I t]$ is $\mathrm{C}-\mathrm{M}$, it suffices by Remark 1.12 to show that $(R[I t] /(a t))_{(M, I t)}$ is C-M. By Remark 1.9 and the above exact sequence, this will hold if both $(a, a t) /(a t)$ and $R[I t] /(a, a t)$ are C-M. We have that $(a, a t) /(a t) \cong(a) /(a) \cap(a t)=(a) / a(a t: a) \cong R[I t] /(a t: a)$, the last isomorphism using that $a$ is an $R[I t]$-regular element. It follows easily that $(a t: a)=(I t)$, thus $(a, a t) /(a t) \cong R[I t] /(I t)=R$ is C-M. As $a^{\prime}$ is a $\operatorname{gr}(I, R)$ regular element and $a \in I \backslash I^{2}$, we have that $R[I t] /(a, a t)=R_{1}\left[I_{1} t\right]$ (see the proof of [Hucl, Proposition 2.1]) which is C-M by assumption. This completes the proof.

Another piece of information we will need for our induction arguments is a description of $l(I /(f))$ where $f$ is an element of the ideal $I$. In particular, we need to know that if $f \in I$ and $f^{\prime}$ is a $\operatorname{gr}(I, R)$-regular element then $l(I /(f)) \geq l(I)-1$. We also need to use that if $x \in R \backslash I$ and $x^{\prime}$ is a $\operatorname{gr}(I, R)$ regular element, then $l(I)=l((I, x) /(x))$. These technical statements have been worked out in [Huc1, Huc2] and the next lemma summarizes them.

Lemma 2.12. Let $(R, M)$ be a local ring and $I$ an ideal of $R$.

(i) If $f \in I$ and $f^{\prime}$ is a $\operatorname{gr}(I, R)$-regular element, then $l(I /(f)) \geq l(I)-1$.

(ii) If $x \in M \backslash I$ and $x^{\prime}$ is a $\operatorname{gr}(I, R)$-regular element, then $l((I, x) /(x))=$ $l(I)$.

Proof. (i) This is essentially [Huc2, Proposition 2.2(i)], the difference being that there $R$ was assumed to be an integral domain, $I$ was assumed to be a prime ideal, and $f$ was assumed to be superficial for $I$. If $f^{\prime}$ is a $\operatorname{gr}(I, R)$ regular element, then $f$ is superficial for $I$ however. Also, the proof of [Huc2, Proposition 2.2(i)] goes through for any local ring $R$ and any ideal $I$ of $R$.

(ii) If $x \notin I$ and $x^{\prime}$ is a $\operatorname{gr}(I, R)$-regular element then it is easy to see that if $R_{1}=R /(x)$ and $I_{1}=(I, x) /(x)$ then $R_{1}\left[I_{1} t\right] \cong R[I t] /(x)$ (see for example [Huc1, Proposition 2.1]). This isomorphism implies that $R[I t] / M R[I t] \cong$ $R_{1}\left[I_{1} t\right] / M_{1} R_{1}\left[I_{1} t\right]$ where $M_{1}=M /(x)$. Therefore $l(I)=l((I, x) /(x))$.

Finally we discuss the well-known technique of passing from $R$ to $R[X]_{M[X]}$ in order to reduce to the case of an infinite residue field. As mentioned in the introduction, when $R / M$ is infinite the concept of an ideal having analytic deviation $k$ is closely related to that of having deviation $k$. This is true because a minimal reduction of an analytic deviation $k$ ideal has deviation $k$ if $R / M$ is infinite. The following lemma, all of which has appeared in the literature, shows that most of our assumptions carry through under passage to the ring $R(X)$, thus allowing a reduction to the infinite residue field case some of the time.

Lemma 2.13. Let $(R, M)$ be a local ring, $X$ an indeterminant, and $R(X)=$ $R[X]_{M[X]}$. Let $I$ be an ideal of $R$ and let $I(X)$ denote the ideal $I R(X)$. Then

(i) $\operatorname{ht}(I)=\operatorname{ht}(I(X))$,

(ii) $l(I)=l(I(X))$,

(iii) $\operatorname{gr}(I, R)$ is $C-M$ if and only if $\operatorname{gr}(I(X), R(X))$ is $C-M$,

(iv) $R[I t]$ is $C-M$ if and only if $R(X)[I(X) t]$ is $C-M$,

(v) If $P_{Q}$ is a complete intersection for all $Q$ containing $P$ such that ht $(Q / P)=k$, then the same statement holds for $P(X)$. 
Proof. (i) and (ii) were proved in [HO, Lemma 1]. Parts (iii) and (iv) were proved in [GS1, Lemma 3.8], and follow from the observations that

$$
\operatorname{gr}(I(X), R(X))=\operatorname{gr}(I, R)[X]_{S}
$$

where

$$
S=(R[X] / I[X]) \backslash(M[X] / I[X])
$$

and $R(X)[I(X) t]=R[I t][X]_{U}$ where $U=R[X] \backslash M[X]$. To prove (v) let $q$ be a prime ideal of $R(X)$ that contains $P(X)$ and assume that $\mathrm{ht}(q / P(X))=k$. Set $Q=q \cap R$ and $S=R \backslash Q$. Then $P_{Q}$ is a complete intersection because ht $(Q / P) \leq k$, hence $P(X)_{S}$ is a complete intersection. But $P(X)_{q}$ is a further localization of $P(X)_{S}$, therefore $P(X)_{q}$ is also a complete intersection.

Remark 2.14. The only property that we need which does not pass cleanly to the ring $R(X)$ is that of $I$ having $r(I) \leq 1$. For example let

$$
R=k[[Y, Z]] /(Y Z(Y+Z))
$$

where $k=\mathbb{Z} / 2 \mathbb{Z}$ and let $I=(Y, Z) R$. Then $I$ has no proper reduction hence $r(I)=0$. On the other hand $r(I(X))=2$. This ideal $I$ is the "standard" example of one whose minimal reductions are not generated by $l(I)$ elements. If $R / M$ is assumed to be finite in Theorem 2.1 , then the conclusion will still hold provided we assume that $r(I(X)) \leq 1$ (instead of $r(I) \leq 1)$. This follows by applying Lemma 2.13 .

Proof of Theorem 2.1. Note that (i) and (ii) are equivalent by [HH, Theorem 2.9], and it follows from [Hun 1, Proposition 1.1] that (iii) implies (ii). We will prove that (i) implies (iii). The proof is by induction on the height of $I$. Suppose $\operatorname{ht}(I)=1$ and $l(I)=2$. Let $J$ be a minimal reduction of $I$ such that $J I=I^{2}$, and choose a generating set $\left\{a_{1}, c\right\}$ for $J$ satisfying the conditions that each is a regular element and $\left(a_{1}\right)$ generates $I$ generically. We will show that $R[I t]$ is C-M by induction on $\operatorname{dim}(R / I)$ (note that necessarily we have $\operatorname{dim}(R / I)>0)$. Suppose $\operatorname{dim}(R / I)=1$, and let $y \in M$ be such that $\left\{a_{1}, y\right\}$ is a regular sequence. Since $\operatorname{dim}\left(R[I t]_{(M, I t)}\right)=3$, it suffices by Remark 1.12 to show that $\operatorname{grade}(M, I t)=3$. We claim that $\left\{a_{1} t, a_{1}+c t, y\right\}$ is an $R[I t]$ regular sequence. Since $a_{1}$ is an $R$-regular element, $a_{1} t$ is an $R[I t]$-regular element. Suppose $\left(u_{0}+u_{1} t+\cdots+u_{n} t^{n}\right)\left(a_{1}+c t\right) \in\left(a_{1} t\right)$. Then

$$
\left(u_{0}+u_{1} t+\cdots+u_{n} t^{n}\right)\left(a_{1}+c t\right)=\left(v_{0}+v_{1} t+\cdots+v_{m} t^{m}\right) a_{1} t
$$

for some $v_{i} \in I^{i}$. We may assume that $m \geq n$ by adding zeros if necessary. We first reduce to the case $n=0$. From the relation we have that $c u_{n}=a_{1} v_{n}$. If $n \geq 1$, this implies that $u_{n} \in\left(a_{1}: c\right) \cap I$. Thus $u_{n} \in\left(a_{1}\right)$ by Lemma 2.5 . It then follows from Lemma 2.6(ii) that $u_{n} \in a_{1} I^{n-1}$, hence $u_{n}=a_{1} r$ where $r \in I^{n-1}$. Therefore $u_{n} t^{n}=\left(a_{1} t\right) r t^{n-1} \in\left(a_{1} t\right)$, and it follows that $\sum_{i=0}^{n} u_{i} t^{i} \in\left(a_{1} t\right)$ if and only if $\sum_{i=0}^{n-1} u_{i} t^{i} \in\left(a_{1} t\right)$. This reduces the problem to the case $n=0$. Write $u_{0}\left(a_{1}+c t\right)=\left(v_{0}+v_{1} t+\cdots+v_{m} t^{m}\right)\left(a_{1} t\right)$. Then $a_{1} u_{0}=0$, thus $u_{0}=0$. This shows that $\left\{a_{1} t, a_{1}+c t\right\}$ is a regular sequence.

Suppose that $\left(u_{0}+u_{1} t+\cdots+u_{n} t^{n}\right) y \in\left(a_{1} t, a_{1}+c t\right)$. Then

$$
\begin{aligned}
\left(u_{0}+u_{1} t+\cdots+u_{n} t^{n}\right) y= & \left(v_{0}+v_{1} t+\cdots+v_{m} t^{m}\right) a_{1} t \\
& +\left(w_{0}+w_{1} t+\cdots+w_{m} t^{m}\right)\left(a_{1}+c t\right)
\end{aligned}
$$


where $m \geq n$ (add zeros on the right-hand side if necessary). Once again we first reduce to the case $n=0$. If $n \geq 2$, then $u_{n} \in I^{n}=\left(a_{1}, c\right) I^{n-1}$ because $r(I) \leq 1$. Write $u_{n}=a_{1} s_{1}+c s_{2}$ where $s_{1}, s_{2} \in I^{n-1}$. Then we have that $u_{n} t^{n}=a_{1} s_{1} t^{n}+c s_{2} t^{n}=\left(a_{1} t\right)\left(s_{1} t^{n-1}-s_{2} t^{n-2}\right)+\left(a_{1}+c t\right)\left(s_{2} t^{n-1}\right) \in\left(a_{1} t, a_{1}+c t\right)$.

This reduces to $n=1$. If $n=1$, then from (2.15) we have that

$$
y u_{1}=a_{1} v_{0}+a_{1} w_{1}+c w_{0}
$$

and

$$
y u_{0}=a_{1} w_{0} .
$$

From (2.17), there exists $r$ such that $u_{0}=a_{1} r$ and $w_{0}=y r$, because $\left\{a_{1}, y\right\}$ is an $R$-sequence. Substituting into (2.16) yields $y\left(u_{1}-c r\right)=a_{1}\left(v_{0}+w_{1}\right)$, hence there exists $s$ such that $u_{1}-c r=a_{1} s$. Therefore $u_{1} t=\left(a_{1} s+c r\right) t=$ $\left(a_{1} t\right) s+\left(a_{1}+c t\right) r-a_{1} r$, which reduces to $n=0$. If $n=0$, then we obtain from (2.15) that

$$
0=a_{1}\left(v_{0}+w_{1}\right)+c w_{0}
$$

and

$$
y u_{0}=a_{1} w_{0} .
$$

From (2.19) there exists $r$ such that $u_{0}=a_{1} r$ and $w_{0}=y r$. Substituting into (2.18) yields that $0=a_{1}\left(v_{0}+w_{1}\right)+y(c r)$. Thus, there exists $s$ such that $c r=a_{1} s$. Therefore $u_{0}=a_{1} r=r\left(a_{1}+c t\right)-\left(a_{1} t\right) s \in\left(a_{1} t, a_{1}+c t\right)$. This completes the proof of the claim that $\left\{a_{1} t, a_{1}+c t, y\right\}$ is a regular sequence, and hence proves that $R[I t]$ is C-M if $\mathrm{ht}(I)=1$ and $\operatorname{dim}(R / I)=1$.

Suppose that $\operatorname{dim}(R / I)=m>1$, and assume that $R[A t]$ is C-M if ht $A=$ $1, \operatorname{dim}(R / A)<m$, and $A$ satisfies the hypotheses of the theorem. For our inductive step, we will use an argument that is very similar to that found in [HH, Theorem 2.9]. Let

$$
\begin{aligned}
\mathscr{A}= & \left\{Q \in \operatorname{Spec}(R) \mid I_{Q} \text { is not a complete intersection }\right\} \\
& \cap\{Q \in \operatorname{Spec}(R) \mid Q \supset I \text { and } \operatorname{ht}(Q / I)=1\} .
\end{aligned}
$$

Since $I$ is generically a complete intersection, $\mathscr{A}$ is a finite set (we are using the fact that the first collection in the intersection is a Zariski-closed set; see [Sw, Remark 2]). By Lemma 2.6(i) it follows that

$$
\operatorname{depth}\left(R /\left(\left(a_{1}\right)+I^{n}\right)\right) \geq \min \{\operatorname{depth}(R / I), \operatorname{dim}(R / I)-1\} \text { for all } n \geq 1 .
$$

Since $\operatorname{depth}(R / I) \geq \operatorname{dim}(R / I)-1$ by assumption, we find that

$$
\operatorname{depth}\left(R /\left(\left(a_{1}\right)+I^{n}\right)\right) \geq \operatorname{dim}(R / I)-1 \geq 1 .
$$

By Lemma 2.8 there exists an element $x \in M$ such that $x^{\prime}$ is a $\operatorname{gr}(I, R)$-regular element and $x \notin Q$ if $Q \in \mathscr{A}$. In particular, $x$ is an $R$-regular element.

Set $I_{1}=(I, x) /(x)$ and $R_{1}=R /(x)$. Then $\operatorname{ht}\left(I_{1}\right)=\mathrm{ht}(I)=1$ and $\operatorname{depth}\left(R_{1} / I_{1}\right) \geq \operatorname{dim}\left(R_{1} / I_{1}\right)-1$. Also $l\left(I_{1}\right)=l(I)=2$ (by Lemma 2.12), hence $J_{1}=(J, x) /(x)$ is a minimal reduction of $I_{1}$. Thus $r\left(I_{1}\right) \leq r(I) \leq 1$. Furthermore, $I_{1}$ is generically a complete intersection since $x \notin Q$ if $I_{Q}$ is not a complete intersection and $\operatorname{ht}(Q)=2$ (by our choice of $x$ ). Therefore the induction hypothesis applies to the pair $\left(R_{1}, I_{1}\right)$ to show that $R_{1}\left[I_{1} t\right]$ is 
C-M. Since $x^{\prime}$ is a $\operatorname{gr}(I, R)$-regular element we have that $R_{1}\left[I_{1} t\right] \cong R[I t] /(x)$, hence $R[I t] /(x)$ is C-M. This implies that $R[I t]$ is C-M.

We now continue with our induction on $h t(I)$. Suppose $h t(I)=d>1$ and assume the theorem is true for ideals having height at most $d-1$. Let $J$ be a minimal reduction of $I$ satisfying $J I=I^{2}$, and assume $J=\left(a_{1}, \ldots, a_{d}, c\right)$ where $a_{1}, \ldots, a_{d}, c$ are chosen so that $\left\{a_{1}, \ldots, a_{d}\right\}$ is a regular sequence that generates $I$ generically, and $c$ is a regular element (we can do this by using a general position argument). Since $\left(a_{1}, \ldots, a_{d}\right) \cap I^{n}=\left(a_{1}, \ldots, a_{d}\right) I^{n-1}$ by Lemma 2.6(ii), it follows from Remark 1.11 that $a_{1}^{\prime}$ is a $\operatorname{gr}(I, R)$-regular element.

Let $R_{1}=R /\left(a_{1}\right)$ and $I_{1}=I /\left(a_{1}\right)$. Since $a_{1}$ is a part of a minimal generating set for $I$ locally at $\min (R / I), I_{1}$ is generically a complete intersection. Note that $\operatorname{ht}\left(I_{1}\right)=\operatorname{ht}(I)-1 \geq 1$. Since $l\left(I_{1}\right)=l(I)-1$ (by Lemma 2.12) it follows that $\operatorname{ad}\left(I_{1}\right)=1$. Thus $J_{1}=J /\left(a_{1}\right)$ is a minimal reduction of $I_{1}$ such that $J_{1} I_{1}=I_{1}^{2}$, hence $r\left(I_{1}\right) \leq 1$. Therefore the induction hypotheses are satisfied for $R_{1}$ and $I_{1}$ so that $R_{1}\left[I_{1} t\right]$ is C-M. We now apply Lemma 2.11 to obtain that $R[I t]$ is C-M thus finishing the proof of Theorem 2.1.

We list several corollaries to Theorem 2.1.

Corollary 2.20. Let $(R, M)$ be a $C$-M local ring with $R / M$ infinite and let $I$ be an ideal of $R$ such that $\operatorname{ad}(I)=1, \mathrm{ht}(I) \geq 1$, the minimal primes of $R / I$ have the same height, the associated primes of $R / I$ have height at most $\mathrm{ht}(I)+1$ and $I$ is generically a complete intersection. Assume also that there exists a minimal reduction $J$ of $I$ such that $r_{J}\left(I_{Q}\right) \leq 1$ for every prime ideal $Q \supset I$ with $\mathrm{ht}(Q / I)=1$. Then the following are equivalent.

(i) $\operatorname{depth}(R / I) \geq \operatorname{dim}(R / I)-1$,

(ii) $\operatorname{gr}(I, R)$ is $C-M$,

(iii) $R[I t]$ is $C$-M.

Proof. It follows from [HH, Theorem 2.2] that $r(I) \leq 1$. Now use Theorem 2.1.

Corollary 2.21. Let $R$ be a $C-M$ local ring and $P$ a prime ideal of $R$. Assume that $\operatorname{ad}(P)=1, \operatorname{ht}(P) \geq 1$ and $P_{Q}$ is a complete intersection for every $Q \supset P$ with $\operatorname{ht}(Q / P)=1$. Then the following are equivalent.

(i) $\operatorname{depth}(R / P) \geq \operatorname{dim}(R / P)-1$,

(ii) $\operatorname{gr}(P, R)$ is $C$ - $M$,

(iii) $R[P t]$ is $C-M$.

Proof. After observing that $\operatorname{depth}(R / P)=\operatorname{depth}(R(X) / P(X))$ and $\operatorname{dim}(R / P)$ $=\operatorname{dim}(R(X) / P(X))$ we are able to use Lemma 2.13 to reduce to the case where $R / M$ is infinite. The result now follows from Corollary 2.20 .

With the next corollary, we place Corollary 2.21 into a less general setting.

Corollary 2.22. Let $R$ be a regular local ring and $P$ a prime ideal of $R$. Assume that $\operatorname{ad}(P)=1$ and $R / P$ is nonsingular in codimension 1 . Then the following are equivalent.

(i) $\operatorname{depth}(R / P) \geq \operatorname{dim}(R / P)-1$,

(ii) $\operatorname{gr}(P, R)$ is $C$ - $M$,

(iii) $R[P t]$ is $C-M$. 
Proof. Follows from Corollary 2.21.

We saw in [HH, Example 4.7] that if $P$ is a prime ideal of a regular local ring $R$ whose powers and symbolic powers coincide, then it need not follow that $R[P t]$ is $\mathrm{C}-\mathrm{M}$. This example was produced in response to [BSV, Question 2.13 , as well as to show that the C-M property of the Rees algebra is a stronger hypothesis than one needs in order to obtain the equivalence " $P^{n}=P^{(n)}$ for all $n \geq 1$ if and only if $l\left(P_{Q}\right)<\mathrm{ht}(Q)$ for all primes $Q$ properly containing $P$ ". As mentioned in the introduction (see (1.2)), if $R[P t]$ is C-M then this equivalence was known to hold. One feature of the results in [HH] was that the C-M assumption on $R[P t]$ was not required, and [HH, Example 4.7] gave an example to which [HH, Corollary 2.7] applied but $R[P t]$ was not C-M. The next application shows that if $P$ has dimension 2 , then the C-M property of $R[P t]$ is forced if $P^{n}=P^{(n)}$ for all $n \geq 1$. In other words, examples such as [HH, Example 4.7] cannot exist if $\operatorname{dim}(R / P)=2$. The key property that prohibits that existence is that one always has $\operatorname{depth}(R / P) \geq \operatorname{dim}(R / P)-1$ if $\operatorname{dim}(R / P)=2$.

Corollary 2.23. Let $R$ be a regular local ring and $P$ a prime ideal of $R$ such that $\operatorname{dim}(R / P)=2$. If $P^{n}=P^{(n)}$ for all $n \geq 1$, then $R[P t]$ is $C-M$.

Proof. Since $\operatorname{dim}(R / P)=2$ we have that $\operatorname{depth}(R / P) \geq \operatorname{dim}(R / P)-1$. As $P^{n}=P^{(n)}$ for all $n \geq 1$, it follows from Burch's inequality $(1.1)$ that $l\left(P_{Q}\right)<$ $\mathrm{ht}(Q)$ for all $Q$ properly containing $P$. Therefore $l(P) \leq \mathrm{ht}(P)+1$. If $l(P)=\operatorname{ht}(P)$, then $P$ is a complete intersection by Theorem 1.3 , hence $R[P t]$ is C-M by [Ba]. If $l(P)=\operatorname{ht}(P)+1$, then $P$ has analytic deviation 1 , and since $l\left(P_{Q}\right)=\operatorname{ht}(P)$ for $Q \supset P$ with $\operatorname{ht}(Q / P)=1, P_{Q}$ is a complete intersection for all $Q \supset P$ with $h t(Q / P)=1$ by Theorem 1.3. Therefore $R[P t]$ is C-M by Corollary 2.21 .

Next, we make an observation about minimal reductions of dimension 1 primes of a regular local ring.

Corollary 2.24. Let $R$ be a regular local ring and $P$ a prime ideal of $R$ such that $\operatorname{dim}(R / P)=1$. Let $I$ be a minimal reduction of $P$. Then $R[I t]$ is $C-M$. Proof. By passing to $R(X)$ we may assume that $R / M$ is infinite. If $P$ is a complete intersection then $I=P$ and $R[I t]$ is $\mathrm{C}-\mathrm{M}$ by [Ba]. If not, then $\operatorname{ad}(P)=1$ by Theorem 1.3 , hence $\operatorname{ad}(I)=1$. Since $I$ is its own minimal reduction we have $r(I)=0$, and since $\operatorname{dim}(R / I)=1$ we have that $\operatorname{depth}(R / I) \geq \operatorname{dim}(R / I)-1$. Finally, since $I R_{P}=P R_{P}$ (because $R_{P}$ is a regular local ring) and $\sqrt{I}=P, I$ is generically a complete intersection. The corollary now follows from Theorem 2.1 .

Corollary 2.24 is of interest to us because recent evidence suggests that $R[P t]$ need not be C-M if $R$ is a regular local ring and $P$ is a prime ideal of $R$ such that $\operatorname{dim}(R / P)=1$ (although we do not yet know an example). We find it interesting that, on the other hand, every minimal reduction of $P$ has a C-M Rees algebra if $\operatorname{dim}(R / P)=1$.

We end this section with an example that is lifted from [ $\mathrm{HH}$, PropositionExample 4.1]. That family of examples was a prime motivator for the work in $[\mathrm{HH}]$ and is also illustrative of the results of this section. Special cases of it have also been studied in [MS and Sc]. 
Example 2.25. Let $P$ be a prime ideal of $T=K[U, V, W, Z]$ where $K$ is a field. Assume that $\operatorname{ht}(P)=2$ and $U W-V Z \in P$. Then $T[P t]$ is C-M. Consequently $T[P t]$ is Gorenstein by [ST, Corollary 3.4].

Proof. This follows from Corollary 2.23 because by [HH, Example-Proposition 4.1(i)] we have that $P^{n}=P^{(n)}$ for all $n \geq 1$, and it suffices to prove the result locally at $(U, V, W, Z)$.

\section{Preliminaries for analytic deViation 2}

Our goal in $\S \S 3$ and 4 is to study the C-M property of the Rees algebra of an analytic deviation 2 ideal. Our assumptions on $R$ and $I$ will be more restrictive than those in $\S 1$. As in [HH, $\S 3]$, the analytic deviation 2 case is more difficult than is the analytic deviation 1 case. In particular, extra care must be taken in choosing a generating set for a minimal reduction of $I$. This section is a technical one whose purpose is to show how we will choose such a generating set, and to display some of the useful properties of such a choice. The reader may find it easier to start with $\S 4$ and refer back to this section as needed.

Our proofs in this section will require some background material. In particular we will make use of a result from basic element theory. If $R$ is a noetherian ring, $N$ is a finitely generated $R$-module, and $P$ is a prime ideal of $R$, then an element $x \in N$ is said to be basic for $N$ at $P$ if $x$ is part of a minimal generating set for $N_{P}$. If $\mathscr{P}$ is a collection of prime ideals, then we say that $x$ is basic for $N$ at $\mathscr{P}$ if $x$ is basic for $N$ at $P$ for every $P \in \mathscr{P}$. If $\mathscr{P}$ is a collection of prime ideals and $P \in \mathscr{P}$, then let $\operatorname{dim}_{\mathscr{P}}(P)$ denote the length of a maximal chain of primes containing $P$ and belonging to $\mathscr{P}$. We will use the following theorem, which is a special case of a result due to Eisenbud and Evans.

Theorem A [EE, Theorem A, SW, Theorem 3.5]. Let $R$ be a commutative noetherian ring and $M$ a finitely generated $R$-module. Let $\mathscr{P}$ be a collection of prime ideals of $R$ and set $d=\max \left\{\operatorname{dim}_{\mathscr{P}}(P) \mid P \in \mathscr{P}\right\}$. Assume that if $P_{\alpha} \in \mathscr{P}$ and $P=\bigcap_{\alpha} P_{\alpha}$, then $P \in \mathscr{P}$. If $\mu\left(M_{P}\right) \geq d+1$ for every $P \in \mathscr{P}$, then there exists an element $x \in M$ that is basic for $M$ at $\mathscr{P}$.

Remark 3.1. By using Flenner's result [Fl, Satz 1.5], we can also assume (if $R$ is local and has an infinite residue field) that $x$ is a minimal generator of $M$ (see [HH, Remark 2]).

Remark 3.2. Let $I$ be an ideal of a commutative noetherian ring $R$ and let $P_{1}, \ldots, P_{n} \in \operatorname{Spec}(R)$. Assume that $R / P_{i}$ is infinite for each $i$, and that $I_{P_{i}} \neq(0)$ for each $i$. Then there exists an element $x \in I$ that is basic for $I$ at $P_{1}, \ldots, P_{n}$.

Proof. See [HH, Remark 2].

We now prove some technical results. The first proposition and the lemma that follows it show how to choose a useful generating set of a minimal reduction of an analytic deviation 2 ideal. The second proposition, whose proof will require two more lemmas, isolates a key property enjoyed by this particular choice of a minimal reduction. 
Proposition 3.3. Let $(R, M)$ be a Gorenstein local ring and $I$ an analytic deviation 2 ideal having height at least 2 . Assume that $R / M$ is infinite, $I_{Q}$ is a complete intersection for all primes $Q \supset I$ such that $\mathrm{ht}(Q / I)=1$ and $R / I$ is $C-M$. Let $s=\mathrm{ht}(I)$ and let $J$ be a minimal reduction of $I$. Assume that $J I=I^{2}$. Then there exists a generating set $\left\{a_{1}, \ldots, a_{s}, c, d\right\}$ for $J$ satisfying the following properties:

(i) $\left\{a_{1}, \ldots, a_{s}\right\}$ is a regular sequence that generates $I$ generically, and $a_{1}$ is basic for $J$ at $\mathscr{P}=\{Q \in \operatorname{Spec}(R) \mid Q \supseteq I$ and $\mathrm{ht}(Q / I) \leq 1\}$

(ii) $\operatorname{ht}\left(\left(a_{1}, \ldots, a_{s}, c\right): I\right) \geq s+1$;

(iii) $\mathrm{ht}\left[\left(\left(a_{1}, \ldots, a_{s}, c\right): I\right)+I\right] \geq s+2$;

(iv) $\left(\left(a_{1}, \ldots, a_{s}\right): c\right)=\left(\left(a_{1}, \ldots, a_{s}\right): d\right)=\left(\left(a_{1}, \ldots, a_{s}\right): I\right)$;

(v) $\left(\left(a_{1}, \ldots, a_{s}\right): c\right) \cap I=\left(a_{1}, \ldots, a_{s}\right)$;

(vi) $\left(\left(a_{1}, \ldots, a_{s}, c\right): d\right) \cap I=\left(a_{1}, \ldots, a_{s}, c\right)$;

(vii) $\left(a_{1}, \ldots, a_{s}, c\right) \cap I^{n}=\left(a_{1}, \ldots, a_{s}, c\right) I^{n-1}$ for all $n \geq 1$;

(viii) $\left(a_{1}, \ldots, a_{s}\right) \cap I^{n}=\left(a_{1}, \ldots, a_{s}\right) I^{n-1}$ for all $n \geq 1$.

Proof. Let $\mathscr{P}=\{Q \in \operatorname{Spec}(R) \mid Q \supset I$ and $\mathrm{ht}(Q / I) \leq 1\}$. Since $I_{Q}$ is a complete intersection for all $Q \in \mathscr{P}$, we have that $J_{Q}=I_{Q}$, hence $\mu\left(J_{Q}\right)=$ $s \geq 2$ for all $Q \in \mathscr{P}$. Therefore, by Theorem A there exists $a_{1} \in J$ that is basic for $J$ at $\mathscr{P}$. Since $J I$ is not contained in any associated prime of $R$, we can choose by using Remark 1.10 an element $a_{1}+y \in a_{1}+J I$ such that $a_{1}+y$ is a regular element of $R$. Since $a_{1}+y$ is still basic for $J$ at $\mathscr{P}$, we can replace $a_{1}$ with $a_{1}+y$ and thus assume that $a_{1}$ is $R$-regular. Furthermore, by using Remark 3.1 we may also assume that $a_{1}$ is a minimal generator of $J$. If $s \geq 3$, then we may repeat this argument to choose $a_{2} \in J$ such that the image of $a_{2}$ in $R /\left(a_{1}\right)$ is basic for $J /\left(a_{1}\right)$ at $\mathscr{P}, a_{2}$ is a minimal generator of $J /\left(a_{1}\right)$, and $a_{2}$ is regular on $R /\left(a_{1}\right)$. Continuing in this fashion we choose $a_{1}, \ldots, a_{s-1} \in J$ such that $\left\{a_{1}, \ldots, a_{s-1}\right\}$ is a regular sequence that is part of a minimal generating set of $J_{Q}$ for all $Q \in \mathscr{P}$, as well as part of a minimal system of generators for $J$. Next, we choose $a_{s} \in J$ to be a minimal generator of $\left(J /\left(a_{1}, \ldots, a_{s-1}\right)\right)_{P}$ for all $P \in \min (R / I)$. By using Remark 3.1 again we may also assume that $a_{s}$ is a minimal generator of $J /\left(a_{1}, \ldots, a_{s-1}\right)$, and by using a prime avoidance argument similar to the one above, we can arrange it so that $a_{s}$ is regular in $R /\left(a_{1}, \ldots, a_{s-1}\right)$. Thus $\left\{a_{1}, \ldots, a_{s}\right\}$ is a regular sequence that generates $J$, hence $I$, generically. This shows (i), and also implies that $\mathrm{ht}\left[\left(\left(a_{1}, \ldots, a_{s}\right): I\right)+I\right] \geq s+1$.

Let $Q_{1}, \ldots, Q_{n}$ be the height $s+1$ primes of $R$ that contain $\left(\left(a_{1}, \ldots, a_{s}\right)\right.$ : $I)+I$. Note that $\left(\left(a_{1}, \ldots, a_{s}\right): J\right)=\left(\left(a_{1}, \ldots, a_{s}\right): I\right)$, because this equality holds locally at all height $s$ primes, and $\operatorname{Ass}\left(R /\left(\left(a_{1}, \ldots, a_{s}\right): I\right)\right)$ all have height $s$. Thus $\left(\left(a_{1}, \ldots, a_{s}\right): J\right) \subseteq Q_{i}$ for $i=1, \ldots, n$. By Remark 3.2 , there exists $c \in J$ such that the image of $c$ in $R /\left(a_{1}, \ldots, a_{s}\right)$ is basic for $J /\left(a_{1}, \ldots, a_{s}\right)$ at $Q_{1}, \ldots, Q_{n}, M$. Because $\left\{a_{1}, \ldots, a_{s-1}\right\}$ is part of a minimal generating set of $J_{Q_{i}}$ for each $i$ (since $\operatorname{ht}\left(Q_{i} / I\right)=1$ ), and by this choice of $c$, it follows that $\left(a_{1}, \ldots, a_{s-1}, c\right)_{Q_{i}}=J_{Q_{i}}$ for $i=1, \ldots, n$, so in particular $\left(a_{1}, \ldots, a_{s}, c\right)_{Q_{i}}=J_{Q_{i}}$ for $i=1, \ldots, n$. By using prime avoidance we can change $c$ by an element of $J I$ to insure that it in only those height $s$ primes of $\min \left(R /\left(a_{1}, \ldots, a_{s}\right)\right)$ that contain $I$. We claim that $\operatorname{ht}\left(\left(a_{1}, \ldots, a_{s}, c\right): I\right) \geq s+1$. If $\operatorname{ht}(P) \leq s$ and $\left(\left(a_{1}, \ldots, a_{s}, c\right): I\right) \subseteq P$, then $P \in \min \left(R /\left(a_{1}, \ldots, a_{s}\right)\right)$ and $c \in P$, hence $P \in \min (R / I)$, a contradiction 
because $\left(a_{1}, \ldots, a_{s}, c\right)_{P}=I_{p}$. This proves (ii).

We claim that $\mathrm{ht}\left[\left(\left(a_{1}, \ldots, a_{s}, c\right): I\right)+I\right] \geq s+2$. If $\operatorname{ht}(Q) \leq s+1$ and $\left(\left(a_{1}, \ldots, a_{s}, c\right): I\right)+I \subseteq Q$, then $\left(\left(a_{1}, \ldots, a_{s}\right): I\right) \subseteq Q$, hence ht $(Q)=s+1$, thus $Q=Q_{i}$ for some $i$ between 1 and $n$. This implies that $\left(a_{1}, \ldots, a_{s}, c\right)_{Q}$ $=J_{Q}=I_{Q}$, therefore $\left[\left(\left(a_{1}, \ldots, a_{s}, c\right): I\right)+I\right]_{Q}=R_{Q}$, a contradiction. This proves (iii).

Note that our choice of $c$ guarantees that $c$ avoids the set $A=\{P \in$ $\left.\min \left(R /\left(a_{1}, \ldots, a_{s}\right)\right) \backslash \min (R / I)\right\}$. This forces

$$
\left(\left(a_{1}, \ldots, a_{s}\right): c\right)=\left(\left(a_{1}, \ldots, a_{s}\right): I\right),
$$

because the equality holds locally at all $P \in \operatorname{Ass}\left(R /\left(\left(a_{1}, \ldots, a_{s}\right): I\right)\right)$. We now choose $d$ to be a minimal generator of $J /\left(a_{1}, \ldots, a_{s}, c\right)$ and simultaneously to avoid $A$. Then $\left(\left(a_{1}, \ldots, a_{s}\right): d\right)=\left(\left(a_{1}, \ldots, a_{s}\right): I\right)$ which shows (iv).

In order to prove property $(v)$, it suffices to show that

$$
\left(\left(a_{1}, \ldots, a_{s}\right): c\right) \cap I=\left(a_{1}, \ldots, a_{s}\right)
$$

locally at all $Q \in \operatorname{Ass}\left(R /\left(a_{1}, \ldots, a_{s}\right)\right)$. If $Q \in \operatorname{Ass}\left(R /\left(a_{1}, \ldots, a_{s}\right)\right)$, then $\operatorname{ht}(Q)=s$. If $I \subseteq Q$, then $\left(a_{1}, \ldots, a_{s}\right)_{Q}=I_{Q}$ because $Q \in \min (R / I)$, thus

$$
\begin{aligned}
& {\left[\left(\left(a_{1}, \ldots, a_{s}\right): c\right) \cap I\right]_{Q}=\left(\left(\left(a_{1}, \ldots, a_{s}\right): I\right) \cap I\right)_{Q}} \\
& \quad=\left(\left(a_{1}, \ldots, a_{s}\right)_{Q}:\left(a_{1}, \ldots, a_{s}\right)_{Q}\right) \cap\left(a_{1}, \ldots, a_{s}\right)_{Q}=\left(a_{1}, \ldots, a_{s}\right)_{Q} .
\end{aligned}
$$

If $I \nsubseteq Q$, then

$$
\begin{array}{r}
{\left[\left(\left(a_{1}, \ldots, a_{s}\right): c\right) \cap I\right]_{Q}=\left[\left(\left(a_{1}, \ldots, a_{s}\right): I\right) \cap I\right]_{Q}} \\
\quad=\left(\left(a_{1}, \ldots, a_{s}\right)_{Q}: R_{Q}\right) \cap R_{Q}=\left(a_{1}, \ldots, a_{s}\right)_{Q} .
\end{array}
$$

This proves $(\mathbf{v})$.

Set $q=\left(\left(a_{1}, \ldots, a_{s}, c\right): I\right)$ and observe that since $R$ is Gorenstein and $R / I$ is C-M, [KMU, Theorem 6.1(a)] implies that this " $(s+1)$-residual intersection" (see the introduction) is unmixed of height $s+1$. Furthermore,

$$
\left(a_{1}, \ldots, a_{s}, c\right)_{Q}=I_{Q}
$$

if $Q \supseteq I$ and $\operatorname{ht}(Q)=s+1$ (because $\left.\mathrm{ht}\left[\left(\left(a_{1}, \ldots, a_{s}, c\right): I\right)+I\right] \geq s+2\right)$, hence $q$ is a "geometric residual intersection" (see [HU]). Therefore $q \cap I=$ $\left(a_{1}, a_{2}, \ldots, a_{s}, c\right)$ by [KMU, Theorem 6.1(a)]. To prove (vi) it therefore suffices to show that $q=\left(\left(a_{1}, \ldots, a_{s}, c\right): d\right)$. As $q \subseteq\left(\left(a_{1}, \ldots, a_{s}, c\right): d\right)$ we need only show equality locally at $\operatorname{Ass}(R / q)$. Let $Q \in \operatorname{Ass}(R / q)$. Then $\operatorname{ht}(Q)=s+1$ because $R / q$ is unmixed of height $s+1$. Therefore $Q$ does not contain $I$ because $q \subseteq Q$ and ht $(q+I) \geq s+2$. Thus $Q$ does not contain $d$ because the radical of $\left(a_{1}, \ldots, a_{s}, c, d\right)$ is the same as the radical of $I$ and $Q$ must contain $\left(a_{1}, \ldots, a_{s}, c\right)$. Therefore

$$
\left(\left(a_{1}, \ldots, a_{s}, c\right): d\right)_{Q}=\left(\left(a_{1}, \ldots, a_{s}, c\right)_{Q}: d R_{Q}\right)=\left(a_{1}, \ldots, a_{s}, c\right)_{Q}=q_{Q} \text {. }
$$

We prove (vii) by induction on $n$. If $n=1$, then the equality is clear. Let $n>1$ and suppose $r \in\left(a_{1}, \ldots, a_{s}, c\right) \cap I^{n}$. Since $J I=I^{2}$ we have that $J I^{n-1}=I^{n}$ and we can write

$$
r=a_{1} r_{1}+\cdots+a_{s} r_{s}+c r_{s+1}+d r_{s+2} \quad \text { where } r_{i} \in I^{n-1} \text { for } i=1, \ldots, s+2 \text {. }
$$


Thus, $r_{s+2} \in\left(\left(a_{1}, \ldots, a_{s}, c\right): d\right) \cap I=\left(a_{1}, \ldots, a_{s}, c\right)$ by (vi). Hence $r_{s+2} \in$ $\left(a_{1}, \ldots, a_{s}, c\right) \cap I^{n-1}=\left(a_{1}, \ldots, a_{s}, c\right) I^{n-2}$ by the induction hypothesis. Therefore $d r_{s+2} \in\left(a_{1}, \ldots, a_{s}, c\right) I^{n-1}$ and this implies $r \in\left(a_{1}, \ldots, a_{s}, c\right) I^{n-1}$ proving (vii).

We also prove (viii) by induction on $n$, the case $n=1$ being clear. Let $n>1$ and suppose $r \in\left(a_{1}, \ldots, a_{s}\right) \cap I^{n}$. Using (vii) we can write

$$
\begin{aligned}
\left(a_{1}, \ldots, a_{s}\right) \cap I^{n} & =\left(a_{1}, \ldots, a_{s}\right) \cap\left(a_{1}, \ldots, a_{s}, c\right) \cap I^{n} \\
& =\left(a_{1}, \ldots, a_{s}\right) \cap\left(a_{1}, \ldots, a_{s}, c\right) I^{n-1}
\end{aligned}
$$

Thus $r=a_{1} r_{1}+\cdots+a_{s} r_{s}+c r_{s+1}$ where $r_{i} \in I^{n-1}$ for $i=1, \ldots, s+1$. Hence $r_{s+1} \in\left(\left(a_{1}, \ldots, a_{s}\right): c\right) \cap I=\left(a_{1}, \ldots, a_{s}\right)$ by $(v)$. Therefore $r_{s+1} \in$ $\left(a_{1}, \ldots, a_{s}\right) \cap I^{n-1}=\left(a_{1}, \ldots, a_{s}\right) I^{n-2}$ by the induction hypothesis. This implies that $r \in\left(a_{1}, \ldots, a_{s}\right) I^{n-1}$, completing the proof of (viii), and of Proposition 3.3.

Lemma 3.4. Let $R$ and $I$ be as in Proposition 3.3 with $s=2$ and let $\{a, b, c, d\}$ be chosen so as to satisfy the conclusions of Proposition 3.3 (with $a, b$ playing the role of $\left.a_{1}, a_{2}\right)$. Then $c$ and $d$ can be adjusted so that in addition the following are satisfied:

(i) $\{c, d\}$ is an $R$-regular sequence,

(ii) For every prime ideal $Q$ of height 3 either $(a, b)_{Q}=I_{Q}$ or $(c, d)_{Q}=$ $I_{Q}$,

(iii) For all $P \in \min (R / I)$ we have that $(a, b)_{P}=I_{P}=(c, d)_{P}$.

Proof. Let $Q_{1}, \ldots, Q_{n}$ be the height 3 primes that contain $((a, b): I)+I$. Note that this set is finite because $(a, b)_{P}=I_{P}$ for all $P \in \min (R / I)$ by Proposition 3.3(i), hence ht $[((a, b): I)+I] \geq 3$. If $Q$ is a height 3 prime of $R$ and $I \nsubseteq Q$, then $I_{Q}=R_{Q}$. Thus $(a, b)_{Q}=R_{Q}$ or $(c, d)_{Q}=R_{Q}$ because $\sqrt{(a, b, c, d)}=\sqrt{I}$ (no matter how $d$ is chosen). Suppose that $I \subseteq Q$ and $\operatorname{ht}(Q)=3$. If $Q \neq Q_{i}$, then $(a, b)_{Q}=I_{Q}$ because $((a, b): I)+I \nsubseteq Q$. On the other hand, since $\operatorname{ht}[((a, b, c): I)+I] \geq 4$ by Proposition 3.3(iii) we have that $(a, b, c)_{Q_{i}}=I_{Q_{i}}$ for $i=1, \ldots, n$. Also $(a, b)_{Q_{i}} \neq I_{Q_{i}}$ for each $i$, because $((a, b): I)+I \subseteq Q_{i}$, thus $c$ is a minimal generator of $I_{Q_{i}}$ for $i=1, \ldots, n$. Let $J_{i}=\left((c)+J Q_{i}\right)^{e c}$ (the "contraction" back to $R$ of $\left.\left((c)+J Q_{i}\right) R_{Q_{i}}\right)$, and let $A(P)=((c)+J P)^{e c}$ for $P \in \min (R / I)$. We claim that $(d)+(a, b, c J) \nsubseteq J_{i} \cup\left(\bigcup_{P \in \min (R / I)} A(P)\right)$. If $(d)+(c, b, c J) \subseteq J_{i}$, then after localizing at $Q_{i}$ we obtain that $((d)+(a, b, c J))_{Q_{i}} \subseteq\left((c)+J Q_{i}\right)_{Q_{i}}$, hence

$$
J_{Q_{i}}=(a, b, c, d)_{Q_{i}}=((d)+(a, b, c J))_{Q_{i}}+(c)_{Q_{i}} \subseteq\left((c)+J Q_{i}\right)_{Q_{i}},
$$

a contradiction (similarly for $A(P))$. By Remark 1.10, there exists $y \in(a, b, c J)$ such that

$$
d^{\prime}=d+y \notin\left(\bigcup_{i=1}^{n} J_{i}\right) \cup\left(\bigcup_{P \in \min (R / I)} A(P)\right) \text {. }
$$

Clearly $\left(a, b, c, d^{\prime}\right)=J$. Furthermore, after localizing at $Q_{i}, d^{\prime} \notin\left(c, J Q_{i}\right)_{Q_{i}}$ so that $\left(c, d^{\prime}\right)_{Q_{i}}=J_{Q_{i}}$ (we are assuming here that $c$ is a minimal generator of $J_{Q_{i}}$ for each $\left.i\right)$. Also, if $P \in \min (R / I)$ then $d^{\prime} \notin(c, J P)_{P}$. Thus $\left(c, d^{\prime}\right)_{P}=I_{P}$ for all $P \in \min (R / I)$. Finally, we may change $d^{\prime}$ by an element 
of $\bigcap_{P \in \min (R / I)} J P$ without affecting its other properties. This allows us to insure that $\left\{c, d^{\prime}\right\}$ is also a regular sequence.

The next proposition will allow us to make use of Lemma 2.8 in order to perform a crucial inductive step within the proof of Theorem 4.1 (in the next section).

Proposition 3.5. Let $(R, M)$ be a Gorenstein local ring and $I$ an analytic deviation 2 ideal of $R$ having height at least 2 . Assume that $R / I$ is $C-M$ and that $I_{Q}$ is a complete intersection for all $Q \supset I$ such that $\mathrm{ht}(Q / I)=1$. Let $J$ be a minimal reduction of $I$ satisfying $J I=I^{2}$. Assume that $\mathrm{ht}(I)=s$, and $J=\left(a_{1}, \ldots, a_{s}, c, d\right)$ where the generating set is chosen to satisfy the conclusions of Proposition 3.3. Then for all $n \geq 1$,

$$
\left.\operatorname{Ass}\left(R /\left(a_{1}, \ldots, a_{s}\right)+I^{n}\right)\right) \subseteq\{Q \in \operatorname{Spec}(R) \mid \operatorname{ht}(Q) \leq s+2\} .
$$

Proof. The proof is by induction on $s=\mathrm{ht}(I)$. Let $s=2$. For convenience we write $J=(a, b, c, d)$ for the reduction. Using Lemma 3.4 we may assume that $\{c, d\}$ is also an $R$-regular sequence, that for every prime ideal $Q$ of height 3 either $(a, b)_{Q}=I_{Q}$ or $(c, d)_{Q}=I_{Q}$, and for all $P \in \min (k / I)$ we have that $(a, b)_{P}=I_{P}=(c, d)_{P}$.

We will first establish two lemmas in order to handle this base case of $s=2$. These lemmas correspond directly to [HH, Theorem 3.5, Claims 4 and 5].

Lemma 3.6. Let $q=((c, d): I)$. Then $\left((c, d)^{n-1}:(a, b)\right)=(c, d)^{n-2} q$ for all $n \geq 3$.

Proof. It suffices to verify the equality locally at all $Q \in \operatorname{Ass}\left(R /(c, d)^{n-2} q\right)$, because $(c, d)^{n-2} q \subseteq\left((c, d)^{n-1}:(a, b)\right)$. We first isolate these prime ideals. Consider the exact sequence

$$
0 \rightarrow(c, d)^{n-2} /(c, d)^{n-2} q \rightarrow R /(c, d)^{n-2} q \rightarrow R /(c, d)^{n-2} \rightarrow 0 .
$$

Since $(c, d)^{n-1} /(c, d)^{n} \cong[R /(c, d)]^{n}$ we have that

$$
(c, d)^{n-1} /(c, d)^{n-1} q \cong[R /(c, d)]^{n} \otimes_{R} R / q \cong[R / q]^{n} .
$$

Thus from (3.7) it follows that

$$
\operatorname{Ass}\left(R /(c, d)^{n-2} q\right) \subseteq \operatorname{Ass}(R / q) \cup \operatorname{Ass}\left(R /(c, d)^{n-2}\right) \subseteq \operatorname{Ass}(R /(c, d))
$$

by the definition of $q$ and because $R /(c, d)^{n-2}$ is C-M. Let $Q \in \operatorname{Ass}(R /(c, d))$. Note that $q \nsubseteq Q$ if $I \subseteq Q$ because $h t(Q)=2$ and $(c, d)$ generates $I$ generically. If $I \subseteq Q$, then $Q \in \min (R / I)$ hence

$$
\begin{aligned}
\left((c, d)^{n-2} q\right)_{Q} & =(c, d)_{Q}^{n-2}=I_{Q}^{n-2}=\left(I_{Q}^{n-1}: I_{Q}\right) \\
& =\left((c, d)_{Q}^{n-1}:(a, b)_{Q}\right)=\left((c, d)^{n-1}:(a, b)\right)_{Q} .
\end{aligned}
$$

If $I \nsubseteq Q$ then $(a, b) \nsubseteq Q$ because $\sqrt{J}=\sqrt{I}$. Thus

$$
(c, d)_{Q}^{n-2} q_{Q}=(c, d)_{Q}^{n-1}=\left((c, d)_{Q}^{n-1}: R_{Q}\right)=\left((c, d)^{n-1}:(a, b)\right)_{Q} .
$$

This proves Lemma 3.6.

Lemma 3.8. For all $n \geq 1,(a, b) \cap(c, d)^{n-1} I=(a, b)(c, d)^{n-1}$.

Proof. If $n=1$ this is clear, so assume $n \geq 2$. If suffices to show the equality locally at all $Q \in \operatorname{Ass}\left(R /(a, b)(c, d)^{n-1}\right)$. Consider the exact sequence

$$
0 \rightarrow R / A \rightarrow R / a(c, d)^{n-1} \oplus R / b(c, d)^{n-1} \rightarrow R /(a, b)(c, d)^{n-1} \rightarrow 0
$$


where $A=a(c, d)^{n-1} \cap b(c, d)^{n-1}$. Using that $\{a, b\}$ is a regular sequence we find that $A=a b\left[(c, d)^{n-1}:(a, b)\right]$. Thus

$$
\begin{gathered}
\operatorname{Ass}\left(R /(a, b)(c, d)^{n-1}\right) \subseteq \operatorname{Ass}\left(R / a(c, d)^{n-1} \oplus R / b(c, d)^{n-1}\right) \\
\cup\left\{Q \in \operatorname{Spec}(R) \mid \operatorname{depth}\left(R / a b\left[(c, d)^{n-1}:(a, b)\right]\right)_{Q}=1\right\}
\end{gathered}
$$

by Remark 1.9. Using Remark 1.9 and [HH, Remark 2.1(i)] it follows that

$$
\begin{gathered}
\operatorname{Ass}\left(R /(a, b)(c, d)^{n-1}\right) \subseteq \operatorname{Ass}(R /(a)) \cup \operatorname{Ass}(R /(b)) \cup \operatorname{Ass}\left(R /(c, d)^{n-1}\right) \\
\cup\left\{Q \in \operatorname{Spec}(R) \mid \operatorname{depth}\left(R / a b\left[(c, d)^{n-1}:(a, b)\right]\right)_{Q}=1\right\} .
\end{gathered}
$$

If $Q \in \operatorname{Ass}(R /(a)) \cup \operatorname{Ass}(R /(b))$, then $\operatorname{ht}(Q)=1$, hence

$$
\left[(a, b) \cap(c, d)^{n-1} I\right]_{Q}=R_{Q}=\left[(a, b)(c, d)^{n-1}\right]_{Q} .
$$

Suppose $Q \in \operatorname{Ass}\left(R /(c, d)^{n-1}\right)$. If $I \subseteq Q$, then $Q \in \min (R / I)$, hence

$$
\left[(a, b) \cap(c, d)^{n-1} I\right]_{Q}=I_{Q} \cap I_{Q}^{n}=I_{Q}^{n}=\left[(a, b)(c, d)^{n-1}\right]_{Q} .
$$

If $I \nsubseteq Q$, then $(a, b) \nsubseteq Q$, hence

$$
\left[(a, b) \cap(c, d)^{n-1} I\right]_{Q}=\left[\left((c, d)^{n-1}\right]_{Q}=\left[(a, b)(c, d)^{n-1}\right]_{Q} .\right.
$$

Next assume that $\operatorname{depth}\left(R / a b\left[(c, d)^{n-1}:(a, b)\right]\right)_{Q}=1$. By Lemma 3.6,

$$
\left((c, d)^{n-1}:(a, b)\right)=(c, d)^{n-2} q
$$

where $q=((c, d): I)$, hence $\operatorname{depth}\left(R / a b q(c, d)^{n-2}\right)_{Q}=1$. This implies, by [HH, Remark 2.1(i)] that either

$$
\operatorname{depth}(R /(a b))_{Q}=1 \text { or } \operatorname{depth}\left(R / q(c, d)^{n-2}\right)_{Q}=1 .
$$

If $\operatorname{depth}(R /(a b))_{Q}=1$, then $\operatorname{ht}(Q)=2$ and the above argument goes through to show that $(a, b) \cap(c, d)^{n-1} I=(a, b)(c, d)^{n-1}$ locally at $Q$. Suppose that $\operatorname{depth}\left(R / q(c, d)^{n-2}\right)_{Q}=1$. From the exact sequence (3.7), and the isomorphism $(c, d)^{n-2} /(c, d)^{n-2} q \cong[R / q]^{n-1}$, it follows by Remark 1.9 that either $\operatorname{depth}(R / q)_{Q}=1$ or $\operatorname{depth}\left(R /(c, d)^{n-2}\right)_{Q}=1$. Since $R / I$ is C-M and $R$ is Gorenstein, we conclude from Remark 1.8 that $q$ is linked to $I$ and $R / q$ is C-M, thus $\operatorname{ht}(Q)=3$ if $\operatorname{depth}(R / q)_{Q}=1$. Since $R /(c, d)^{n-1}$ is C-M by [Mat, $16 \mathrm{~F}], \operatorname{ht}(Q)=3$ if $\operatorname{depth}\left(R /(c, d)^{n-1}\right)_{Q}=1$. So it suffices then to show the equality locally at height 3 primes. Let $\mathrm{ht}(Q)=3$. By our choice of $a, b, c$, and $d$ we have that either $(a, b)_{Q}=I_{Q}$ or $(c, d)_{Q}=I_{q}$. If $(a, b)_{Q}=I_{Q}$, then

$$
\left[(a, b) \cap(c, d)^{n-1} I\right]_{Q}=I_{Q} \cap(c, d)_{Q}^{n-1} I_{Q}=(c, d)_{Q}^{n-1} I_{Q}=\left[(a, b)(c, d)^{n-1}\right]_{Q} .
$$

If $(c, d)_{Q}=I_{Q}$, then

$$
\begin{aligned}
{[(a, b)} & \left.\cap(c, d)^{n-1} I\right]_{Q} \\
= & \left.(a, b)_{Q} \cap I_{Q}^{n}=\left((a, b) I^{n-1}\right)_{Q} \quad \text { (by Proposition 3.3(viii) }\right) \\
= & {\left[(a, b)(c, d)^{n-1}\right]_{Q} . }
\end{aligned}
$$

This proves Lemma 3.8 . 
We now finish the proof of the height 2 case. For convenience, set $X^{4}=$ $\{Q \in \operatorname{Spec}(R) \mid \mathrm{ht}(Q) \leq 4\}$. Using that $J I=I^{2}$ write $(a, b)+I^{n}=(a, b)+$ $(c, d)^{n-1} I$, and consider the exact sequence

$$
\begin{aligned}
0 & \rightarrow R /\left((a, b) \cap(c, d)^{n-1} I\right) \rightarrow R /(a, b) \oplus R /(c, d)^{n-1} I \\
& \rightarrow R /\left((a, b)+(c, d)^{n-1} I\right) \rightarrow 0 .
\end{aligned}
$$

If $Q \in \operatorname{Ass}\left(R /\left((a, b)+I^{n}\right)\right)$, then either

$$
Q \in \operatorname{Ass}(R /(a, b)) \cup \operatorname{Ass}\left(R /(c, d)^{n-1} I\right)
$$

or

$$
\left.\operatorname{depth}\left[R /(a, b) \cap(c, d)^{n-1} I\right)\right]_{Q}=1 .
$$

If $Q \in \operatorname{Ass}(R /(a, b))$, then $\operatorname{ht}(Q)=2$ so $Q \in X^{4}$. Since $(c, d)^{n-1} /(c, d)^{n} \cong$ $[R /(c, d)]^{n}$ we have that

$$
(c, d)^{n-1} /(c, d)^{n-1} I \cong[R /(c, d)]^{n} \otimes_{R} R / I \cong[R / I]^{n} .
$$

By using the exact sequence $0 \rightarrow(c, d)^{n-1} /(c, d)^{n-1} I \rightarrow R /(c, d)^{n-1} I \rightarrow$ $R /(c, d)^{n-1} \rightarrow 0$ it thus follows that if $Q \in \operatorname{Ass}\left(R /(c, d)^{n-1} I\right)$, then $Q \in$ $\operatorname{Ass}(R / I) \cup \operatorname{Ass}\left(R /(c, d)^{n-1}\right)$. This implies that $\operatorname{ht}(Q)=2$, and we obtain that $Q \in X^{4}$. Suppose that $\operatorname{depth}\left[R /(a, b) \cap(c, d)^{n-1} I\right]_{Q}=1$. By Lemma 3.8 $(a, b) \cap(c, d)^{n-1} I=(a, b)(c, d)^{n-1}$, hence $\operatorname{depth}\left[R /(a, b)(c, d)^{n-1}\right]_{Q}=1$. From the exact sequence (3.9) along with the equality $a(c, d)^{n-1} \cap b(c, d)^{n-1}=$ $a b\left[(c, d)^{n-1}:(a, b)\right]$, it follows from Remark 1.9 that either

$$
\operatorname{depth}\left[R / a(c, d)^{n-1} \oplus R / b(c, d)^{n-1}\right]_{Q} \leq 1
$$

or

$$
\operatorname{depth}\left[R / a b\left((c, d)^{n-1}:(a, b)\right)\right]_{Q} \leq 2 .
$$

If $\operatorname{depth}\left[R / a(c, d)^{n-1} \oplus R / b(c, d)^{n-1}\right]_{Q} \leq 1$, then either $\operatorname{depth}\left[R / a(c, d)^{n-1}\right]_{Q}$ $\leq 1$, or $\operatorname{depth}\left[R / b(c, d)^{n-1}\right]_{Q} \leq 1$. Thus either $\operatorname{depth}(R /(a))_{Q} \leq 1$, $\operatorname{depth}(R /(b))_{Q} \leq 1$, or $\operatorname{depth}\left(R /(c, d)^{n-1}\right)_{Q} \leq 1$ by [HH, Remark 2.1(i)]. In each case, we have that $Q \in X^{4}$. Finally, suppose

$$
\operatorname{depth}\left(R / a b\left[(c, d)^{n-1}:(a, b)\right]\right)_{Q}=2 .
$$

If $n=1$, then $a b\left[\left((c, d)^{n-1}:(a, b)\right]=(a b)\right.$, hence ht $(Q)=3$. If $n \geq 2$, then Lemma 3.6 implies that $a b\left[\left((c, d)^{n-1}:(a, b)\right]=a b q(c, d)^{n-2}\right.$ (where $q=((c, d): I))$, thus $\operatorname{depth}\left[R / a b q(c, d)^{n-2}\right]_{Q}=2$. By [HH, Remark 2.1(i)], it follows that either $\operatorname{depth}[R /(a b)]_{Q} \leq 2$ or $\operatorname{depth}\left[R / q(c, d)^{n-2}\right]_{Q} \leq 2$. In the first case $\operatorname{ht}(Q) \leq 3$, so $Q \in X^{4}$. Suppose that $\operatorname{depth}\left[R / q(c, d)^{n-2}\right]_{Q} \leq 2$. From the exact sequence (3.7), and the isomorphism $(c, d)^{n-2} /(c, d)^{n-2} q \cong$ $[R / q]^{n-1}$, it follows that either $\operatorname{depth}(R / q)_{Q} \leq 2$ or $\operatorname{depth}\left(R /(c, d)^{n-2}\right)_{Q} \leq 2$. Using again that both $R / q$ and $R /(c, d)^{n-1}$ are C-M (by Remark 1.8 and [Mat 16.F]), we obtain that $Q \in X^{4}$. This completes the proof of the proposition if $\mathrm{ht}(I)=2$.

Let $\operatorname{ht}(I)=s>2$ and assume the result is true for ideals of height at most $s-1$. Let $J=\left(a_{1}, \ldots, a_{s}, c, d\right)$ where the generators are chosen to satisfy Proposition 3.3. By Proposition 3.3(i) $a_{1}$ is basic at $\mathscr{P}=\{Q \supseteq$ $I \mid$ ht $(Q / I) \leq 1\}$. Set $R_{1}=R /\left(a_{1}\right), J_{1}=J /\left(a_{1}\right)$, and $I_{1}=I /\left(a_{1}\right)$. Then $\operatorname{ht}\left(I_{1}\right)=s-1 \geq 2, J_{1} I_{1}=I_{1}^{2}$, the images of $a_{2}, \ldots, a_{s}, c, d$ modulo $\left(a_{1}\right)$ 
satisfy the conclusions of Proposition 3.3, and $\left(I_{1}\right)_{Q_{1}}$ is a complete intersection if $Q_{1} \supseteq I_{1}$ and $h t\left(Q_{1} / I_{1}\right)=1$ (because $a_{1}$ is basic at $\mathscr{P}$ ). Furthermore $\operatorname{ad}\left(I_{1}\right)=2$ because $a_{1}^{\prime}$ is a $\operatorname{gr}(I, R)$-regular element by Proposition 3.3(viii) (thus the analytic spread drops by exactly one modulo $\left(a_{1}\right)$ by Lemma 2.12). Therefore the induction hypotheses are satisfied for $R_{1}$ and $I_{1}$. Suppose that $Q \in \operatorname{Ass}\left[R /\left(\left(a_{1}, \ldots, a_{s}\right)+I^{n}\right)\right]$ for some $n \geq 1$. Set $Q_{1}=Q /\left(a_{1}\right)$. Then $Q_{1} \in \operatorname{Ass}_{R_{1}}\left[R_{1} /\left(\left(\overline{a_{1}}, \ldots, \overline{a_{s}}\right)+I_{1}^{n}\right)\right]$ where $\overline{a_{i}}$ is the image of $a_{i}$ modulo $\left(a_{1}\right)$. By the inductive assumption we have that $\operatorname{ht}\left(Q_{1}\right) \leq s+1$. Therefore $\operatorname{ht}(Q) \leq s+2$. This completes the proof of Proposition 3.5.

\section{Analytic deviation 2}

In this section we prove our main result about the C-M property of the Rees algebra of an ideal having analytic deviation 2 . Here is the statement.

Theorem 4.1. Let $(R, M)$ be a Gorenstein local ring such that $R / M$ is infinite and let $I$ be an ideal of $R$ such that $\operatorname{ad}(I)=2$ and $\mathrm{ht}(I) \geq 2$. Assume that $R / I$ is $C-M, r(I) \leq 1$ and $I_{Q}$ is a complete intersection for all $Q \supseteq I$ such that $\operatorname{ht}(Q / I)=1$. Then $R[I t]$ is $C-M$.

Eventually we will prove Theorem 4.1 by induction on $h t(I)$. The following proposition will serve as the base case of the proof, and in many ways its proof contains the most crucial arguments. For us at least, the proof of this proposition displays the best "insight" into why Theorem 4.1 is true.

Proposition 4.2. Let $(R, M)$ be a 4-dimensional Gorenstein local ring such that $R / M$ is infinite and let $I$ be an ideal of $R$ such that $\operatorname{ad}(I)=2$ and $\operatorname{ht}(I)=2$. Assume that $I_{Q}$ is a complete intersection for all $Q$ such that $\operatorname{ht}(Q / I)=1$, $R / I$ is $C-M$, and $r(I) \leq 1$. Then $R[I t]$ is $C-M$.

Proof. Let $J$ be a minimal reduction of $I$ satisfying $J I=I^{2}$. Use Proposition 3.3 to choose a generating set $\{a, b, c, d\}$ for $J$ satisfying the conclusion of that lemma. In order to prove that $R[I t]$ is C-M, it is sufficient by Remark 1.12 to show that $\operatorname{grade}(M, I t)=5$. We claim that $\operatorname{depth}(R /(a, b, c)) \geq 1$.

Consider the exact sequence

$$
0 \rightarrow R /[(a, b) \cap(c)] \rightarrow R /(a, b) \oplus R /(c) \rightarrow R /(a, b, c) \rightarrow 0 .
$$

By Remark 1.9, it follows that either $\operatorname{depth}(R /(a, b, c))=\operatorname{depth}(R /(a, b) \oplus$ $R /(c))$, or $\operatorname{depth}(R /(a, b, c)) \geq \operatorname{depth}[R /((a, b): c) c]-1$. If

$$
\operatorname{depth}(R /(a, b, c))=\operatorname{depth}(R /(a, b) \oplus R /(c)),
$$

then

$$
\operatorname{depth}(R /(a, b, c))=\min \{\operatorname{depth}(R /(a, b)), \operatorname{depth}(R /(c))\}=2 .
$$

This handles the first case. Considering the second case, suppose that

$$
\operatorname{depth}(R /(a, b, c)) \geq \operatorname{depth}[R /((a, b): c) c]-1 .
$$

By [HH, Remark 2.1(i)] we have that

$$
\begin{aligned}
\operatorname{depth}[R /((a, b): c) c] & \geq \min \{\operatorname{depth}(R /(c)), \operatorname{depth}[R /(a, b): c)]\} \\
& =\operatorname{depth}[R /((a, b): c)] .
\end{aligned}
$$

By Proposition 3.3(iv), $((a, b): c)=((a, b): I)$. Hence $R /((a, b): c)$ is C-M by Remark 1.8 (we are using here that $R$ is Gorenstein and $R / I$ is $C-M$ ). Thus 
$\operatorname{depth}[R /((a, b): c)]=2$, hence it follows that $\operatorname{depth}(R /(a, b, c)) \geq 2-1=1$, proving the claim.

Since $\operatorname{depth}(R /(a, b, c)) \geq 1$, we may choose $x$ and $y$ such that $\{a, b$, $x, y\}$ is an $R$-regular sequence, and $((a, b, c): x)=(a, b, c)$. We claim that $\{a t, a+b t, b+c t, x, y+d t\}$ is an $R[I t]$-regular sequence. This will prove that $\operatorname{grade}(M, I t)=5$. To show that $\{a t, a+b t\}$ is a regular sequence, one can use that $\{a, b\}$ is an $R$-regular sequence, and that $(a) \cap I^{n}=a I^{n-1}$ for all $n \geq 1$ (this last condition follows by using induction and Proposition 3.3(viii)). By choice, at is a regular element. Write $\left(u_{0}+u_{1} t+\cdots+u_{n} t^{n}\right)(a+b t)=$ $\left(v_{0}+v_{1} t+\cdots+v_{m} t^{m}\right) a t$ where we may assume that $m \geq n$. Then $b u_{n}=a v_{n}$, hence $u_{n} \in(a) \cap I^{n}=a I^{n-1}$ if $n \geq 1$, thus $u_{n} t^{n} \in(a t)$. This reduces to $n=0$. If $u_{0}(a+b t)=\left(v_{0}+v_{1} t+\cdots+v_{m} t^{m}\right) a t$, then $a u_{0}=0$, hence $u_{0}=0$. This shows that $\{a t, a+b t\}$ is a regular sequence.

Suppose that

$$
\begin{aligned}
& \left(u_{0}+u_{1} t+\cdots+u_{n} t^{n}\right)(b+c t) \\
& \quad=\left(v_{0}+\cdots+v_{m} t^{m}\right) a t+\left(w_{0}+\cdots+w_{m} t^{m}\right)(a+b t),
\end{aligned}
$$

where we may assume (as above) that $m \geq n$. We again reduce to the case $n=0$. From (4.3), we have that $c u_{n}=a\left(v_{n}+w_{n+1}\right)+b w_{n}$ (if $m=n$, then $\left.w_{n+1}=0\right)$. Suppose $n \geq 1$. Then $u_{n} \in((a, b): c) \cap I=(a, b)$ by Proposition 3.3(v), hence $u_{n} \in(a, b) \cap I^{n}=(a, b) I^{n-1}$ by Proposition 3.3(viii). Therefore $u_{n}=a s_{1}+b s_{2}$ where $s_{1}, s_{2} \in I^{n-1}$ and we obtain that $u_{n} t^{n}=$ $s_{1} t^{n-1}(a t)+s_{2} t^{n-1}(a+b t)-a s_{2} t^{n-1}$. Note that modulo $(a t, a+b t)$, and provided that $n \geq 1$, this reduces the degree of $t$ to $n-1$. Therefore we may assume that $n=0$. Suppose that

$$
u_{0}(b+c t)=\left(v_{0}+\cdots+v_{m} t^{m}\right) a t+\left(w_{0}+\cdots+w_{m} t^{m}\right)(a+b t) .
$$

Then $c u_{0}=a\left(v_{0}+w_{1}\right)+b w_{0}$ and $b u_{0}=a w_{0}$. Thus there exists $u$ such that $u_{0}=a u$ and $w_{0}=b u$. Substituting back into the first equation, we get that $a\left(c u-v_{0}-w_{1}\right)=b^{2} u$, hence $u=a r$ for some $r \in R$. Therefore

$$
u_{0}=a^{2} r=a r(a+b t)-b r(a t) \in\{a t, a+b t\},
$$

showing that $\{a t, a+b t, b+c t\}$ is a regular sequence.

Now assume that

$$
\begin{aligned}
& \left(u_{0}+u_{1} t+\cdots+u_{n} t^{n}\right) x \\
& =\left(v_{0}+\cdots+v_{m} t^{m}\right) a t+\left(w_{0}+\cdots+w_{m} t^{m}\right)(a+b t) \\
& \quad+\left(z_{0}+\cdots+z_{m} t^{m}\right)(b+c t) \quad \text { where } m \geq n .
\end{aligned}
$$

Again we will reduce to the $n=0$ case. From (4.4) we obtain that $x u_{n}=$ $a\left(v_{n-1}+w_{n}\right)+b\left(w_{n-1}+z_{n}\right)+c z_{n-1}$, whence $u_{n} \in((a, b, c): x)=(a, b, c)$ by our choice of $x$. If $n \geq 1$, then $u_{n} \in(a, b, c) \cap I^{n}=(a, b, c) I^{n-1}$ by Proposition 3.3(vii), thus $u_{n}=a r_{1}+b r_{2}+c r_{3}$ where $r_{1}, r_{2}, r_{3} \in I^{n-1}$. Therefore

$$
u_{n} t^{n}=r_{1} t^{n-1}(a t)+r_{2} t^{n-1}(a+b t)+r_{3} t^{n-1}(b+c t)-a r_{2} t^{n-1}-b r_{3} t^{n-1} .
$$

As above, but this time modulo ( $a t, a+b t, b+c t)$, this equation reduces $n$. Hence we may assume that $n=0$. From (4.4) we therefore obtain the equations

$$
x u_{0}=a w_{0}+b z_{0}
$$


and

$$
0=a\left(v_{0}+w_{1}\right)+b\left(w_{0}+z_{1}\right)+c z_{0} .
$$

From (4.5) we find that $u_{0} \in(a, b)$ since $\{a, b, x\}$ is a regular sequence. Thus $u_{0}=a r_{1}+b r_{2}$ for some $r_{1}, r_{2} \in R$. Substituting this back into (4.5) yields the equation $a\left(x r_{1}-w_{0}\right)=b\left(z_{0}-x r_{2}\right)$, which implies (since $\{a, b\}$ is a regular sequence) that there exists $r \in R$ such that $z_{0}=x r_{2}+a r$. Substituting this into (4.6) yields the equation $0=a\left(v_{0}+w_{1}+c r\right)+b\left(w_{0}+z_{1}\right)+x\left(c r_{2}\right)$. Since $\{a, b, x\}$ is an $R$-regular sequence, this implies that $c r_{2} \in(a, b)$, say $c r_{2}=a s_{1}+b s_{2}$ for some $s_{1}, s_{2} \in R$. We have then that

$$
\begin{aligned}
u_{0} & =a r_{1}+b r_{2}=a r_{1}+r_{2}(b+c t)-r_{2} c t \\
& =a r_{1}+r_{2}(b+c t)-s_{1}(a t)-s_{2}(a+b t)+a s_{2} .
\end{aligned}
$$

This observation allows us to assume that $u_{0} \in(a)$. Let $u_{0}=a u$ and start over with the relation

$a u x=\left(v_{0}+\cdots+v_{m} t^{m}\right) a t+\left(w_{0}+\cdots+w_{m} t^{m}\right)(a+b t)+\left(z_{0}+\cdots+z_{m} t^{m}\right)(b+c t)$.

Then

$$
\begin{gathered}
a \times u=a w_{0}+b z_{0}, \\
0=a\left(v_{0}+w_{1}\right)+b\left(w_{0}+z_{1}\right)+c z_{0},
\end{gathered}
$$

and

$$
0=a\left(v_{1}+w_{2}\right)+b\left(w_{1}+z_{2}\right)+c z_{1} .
$$

Observe that it suffices to show that $u \in(a, b)$, for then we would have $u=$ $a f_{1}+b f_{2}$ for some $f_{1}, f_{2} \in R$, hence

$$
\begin{aligned}
u_{0} & =a^{2} f_{1}+a b f_{2} \\
& =a f_{1}(a+b t)+a f_{2}(b+c t)-\left(b f_{1}+c f_{2}\right)(a t) \in(a t, a+b t, b+c t) .
\end{aligned}
$$

From (4.7) it follows that $a\left(x u-w_{0}\right)=b z_{0}$, hence there exists $r \in R$ such that $z_{0}=a r$ and $w_{0}=x u-b r$. Substituting into (4.8), we obtain $0=$ $a\left(v_{0}+w_{1}+c r\right)+b\left(x u-b r+z_{1}\right)$, thus $x u-b r+z_{1} \in(a)$. This implies that $x u \in\left(a, b, z_{1}\right)$. From (4.9) we conclude that $z_{1} \in((a, b): c) \cap I$, hence $z_{1} \in(a, b)$ by Proposition 3.3(v). Thus $x u \in(a, b)$, and since $\{a, b, x\}$ is an $R$-regular sequence, this forces $u \in(a, b)$. Therefore we have shown that $\{a t, a+b t, b+c t, x\}$ is a regular sequence.

Finally let

$$
\begin{gathered}
\left(u_{0}+\cdots+u_{n} t^{n}\right)(y+d t)=\left(v_{0}+\cdots+v_{m} t^{m}\right) a t+\left(w_{0}+\cdots+w_{m} t^{m}\right)(a+b t) \\
+\left(z_{0}+\cdots+z_{m} t^{m}\right)(b+c t)+\left(e_{0}+\cdots+e_{m} t^{m}\right) x, \quad \text { where } m \geq n .
\end{gathered}
$$

As before, we will reduce to the case $n=0$. Suppose that $n \geq 1$. From (4.10) we obtain

$$
d u_{n}=a\left(v_{n}+w_{n+1}\right)+b\left(w_{n}+z_{n+1}\right)+c z_{n}+x e_{n+1} .
$$

Since $I^{n+1}=(a, b, c, d) I^{n}$ if $n \geq 1$ and $e_{n+1} \in I^{n+1}$, we may write $e_{n+1}=$ $a r_{1}+b r_{2}+c r_{3}+d r_{4}$ where $r_{i} \in I^{n}$. Substituting this into (4.11) yields that $d\left(u_{n}-x r_{4}\right) \in(a, b, c)$. Therefore $u_{n}-x r_{4} \in((a, b, c): d) \cap I=(a, b, c)$ 
by Proposition 3.3(vi), hence $u_{n}-x r_{4} \in(a, b, c) \cap I^{n}=(a, b, c) I^{n-1}$ by Proposition 3.3(vii). Write $u_{n}=a s_{1}+b s_{2}+c s_{3}+x r_{4}$ where $s_{1}, s_{2}, s_{3} \in I^{n-1}$. Then

$$
\begin{aligned}
u_{n} t^{n}= & a s_{1} t^{n}+b s_{2} t^{n}+c s_{3} t^{n}+x r_{4} t^{n}=s_{1} t^{n-1}(a t)+s_{2} t^{n-1}(a+b t) \\
& +s_{3} t^{n-1}(b+c t)+r_{4} t^{n}(x)-\left(a s_{2} t^{n-1}+b s_{3} t^{n-1}\right),
\end{aligned}
$$

which reduces $n$ (modulo $(a t, a+b t, b+c t, x)$ ). Suppose that $n=0$. Then (4.10) yields the equations

$$
\begin{gathered}
y u_{0}=a w_{0}+b z_{0}+x e_{0}, \\
d u_{0}=a\left(v_{0}+w_{1}\right)+b\left(w_{0}+z_{1}\right)+c z_{0}+x e_{1},
\end{gathered}
$$

and

$$
0=a\left(v_{1}+w_{2}\right)+b\left(w_{1}+z_{2}\right)+c z_{1}+x e_{2} .
$$

We will first use these equations to reduce to the case where $u_{0} \in(a)$. From (4.12) we obtain that $u_{0} \in(a, b, x)$, because $\{a, b, x, y\}$ is an $R$-regular sequence by our choice of $x$ and $y$. Write

$$
u_{0}=a r_{1}+b r_{2}+x r_{3} \quad \text { where } r_{i} \in R \text {. }
$$

Substituting into (4.12) we find that

$$
a\left(y r_{1}-w_{0}\right)+b\left(y r_{2}-z_{0}\right)+x\left(y r_{3}-e_{0}\right)=0,
$$

hence $y r_{1}-w_{0} \in(b, x)$. Write $y r_{1}-w_{0}=b s_{1}+x s_{2}$ where $s_{1}, s_{2} \in R$. Then $w_{0}=y r_{1}-b s_{1}-x s_{2}$, and after substituting this back into (4.16) we obtain $b\left(y r_{2}-z_{0}+a s_{1}\right)=x\left(-y r_{3}+e_{0}-a s_{2}\right)$, thus $y r_{2}-z_{0}+a s_{1}=x s$ for some $s \in R$. We conclude that $z_{0}=y r_{2}+a s_{1}-x s$. Substituting into (4.13) we obtain

$$
a d r_{1}+b d r_{2}+x d r_{3}=a\left(v_{0}+w_{1}+c s_{1}\right)+b\left(w_{0}+z_{1}\right)+x\left(e_{1}-c s\right)+y\left(c r_{2}\right) \text {. }
$$

Thus $c r_{2} \in(a, b, x)$ because $\{a, b, x, y\}$ is an $R$-regular sequence. Write $c r_{2}=a g_{1}+b g_{2}+x g_{3}$ where $g_{i} \in R$. Recall that $x$ was chosen so that $((a, b, c): x)=(a, b, c)$, therefore $g_{3} \in(a, b, c) \subseteq I$. From (4.15) we have that $u_{0}=a r_{1}+b r_{2}+x r_{3}$. Therefore

$$
\begin{aligned}
u_{0} & =a r_{1}+r_{2}(b+c t)+r_{3}(x)-r_{2} c t \\
& =a r_{1}+r_{2}(b+c t)+\left(r_{3}-g_{3} t\right)(x)-g_{1}(a t)-g_{2}(a+b t)+a g_{2} .
\end{aligned}
$$

This equation implies that in order to show $u_{0} \in(a t, a+b t, b+c t, x)$ we may assume that $u_{0} \in(a)$. Set $u_{0}=a u$ and start over as we did in the previous case. Equation (4.10) yields that

$$
\begin{aligned}
a u(y+d t)= & \left(v_{0}+\cdots+v_{m} t^{m}\right) a t+\left(w_{0}+\cdots+w_{m}\right) t^{m}(a+b t) \\
& +\left(z_{0}+\cdots+z_{m} t^{m}\right)(b+c t)+\left(e_{0}+\cdots+e_{m} t^{m}\right) x,
\end{aligned}
$$

from which we obtain

$$
a y u=a w_{0}+b z_{0}+x e_{0}
$$

and

$$
a d u=a\left(v_{0}+w_{1}\right)+b\left(w_{0}+z_{1}\right)+c z_{0}+x e_{1},
$$

$$
0=a\left(v_{1}+w_{2}\right)+b\left(w_{1}+z_{2}\right)+c z_{1}+x e_{2} .
$$


Observe that it suffices to show that $u \in(a, b, x)$, because this would imply that $u=a f_{1}+b f_{2}+x f_{3}$ for some $f_{1}, f_{2}, f_{2} \in R$, hence

$$
\begin{aligned}
u_{0} & =a^{2} f_{1}+a b f_{2}+a x f_{3} \\
& =a f_{1}(a+b t)+a f_{2}(b+c t)+a f_{3}(x)-\left(b f_{1}+c f_{2}\right)(a t) \\
& \in(a t, a+b t, b+c t, x) .
\end{aligned}
$$

From (4.17) it follows that $y u-w_{0} \in(b, x)$, say $y u-w_{0}=b r_{1}+x r_{2}$. Substituting this back into (4.17) yields $b\left(a r_{1}-z_{0}\right)=x\left(e_{0}-a r_{2}\right)$, hence $a r_{1}-z_{0}=x r$ for some $r \in R$. Thus $w_{0}=y u-b r_{1}-x r_{2}$ and $z_{0}=a r_{1}-x r$. Substituting these into (4.18) we find that

$$
a\left(v_{0}+w_{1}-d u+c r_{1}\right)+b\left(y u-b r_{1}+z_{1}\right)+x\left(e_{1}-c r-b r_{2}\right)=0 \text {, }
$$

which implies that $y u-b r_{1}+z_{1} \in(a, x)$. Therefore $y u \in\left(a, b, x, z_{1}\right)$. Since $((a, b, c): x)=(a, b, c)$ it follows from (4.19) that $e_{2} \in(a, b, c)$. Thus $e_{2} \in(a, b, c) I$ by Proposition 3.3(vii). Write $e_{2}=a s_{1}+b s_{2}+c s_{3}$ where $s_{1}, s_{2}, s_{3} \in I$. Then (4.19) implies that

$$
0=a\left(v_{1}+w_{2}+x s_{1}\right)+b\left(w_{1}+z_{2}+x s_{2}\right)+c\left(z_{1}+x s_{3}\right) .
$$

Therefore $z_{1}+x s_{3} \in((a, b): c) \cap I$, so $z_{1}+x s_{3} \in(a, b)$ by Lemma 3.5(v). Thus $z_{1} \in(a, b, x)$ which implies that $y u \in(a, b, x)$. Finally, $u \in(a, b, x)$ since $\{a, b, x, y\}$ is a regular sequence. This concludes the proof that $\{a t, a+$ $b t, b+c t, x, y+d t\}$ is a regular sequence, and therefore finishes the proof of Proposition 4.2.

We can now prove Theorem 4.1. For convenience we restate the result.

Theorem 4.1. Let $(R, M)$ be a Gorenstein local ring such that $R / M$ is infinite and let $I$ be an ideal of $R$ such that $\operatorname{ad}(I)=2$ and $\operatorname{ht}(I) \geq 2$. Assume that $R / I$ is $C-M, r(I) \leq 1$, and $I_{Q}$ is a complete intersection for all $Q \supseteq I$ such that $\operatorname{ht}(Q / I)=1$. Then $R[I t]$ is $C-M$.

Remark. If $R / M$ is assumed to be finite in the statement of Theorem 4.1 , then the theorem will remain true if we add the assumption that $r(I(X)) \leq 1$ (see Lemma 2.13 and Remark 2.14).

Proof. We will prove the theorem by induction on $\mathrm{ht}(I)$. Suppose that $\mathrm{ht}(I)=$ 2 . In order to show that $R[I t]$ is $\mathrm{C}-\mathrm{M}$ in this case, we will use induction on $\operatorname{dim}(R / I)$. If $\operatorname{dim}(R / I)=2$, then Proposition 4.2 states that $R[I t]$ is C-M. Suppose $\operatorname{dim}(R / I)>2$. Let $J$ be a minimal reduction of $I$ satisfying $J I=$ $I^{2}$, and choose a generating set $\left\{a_{1}, a_{2}, c, d\right\}$ of $J$ satisfying the conclusions of Proposition 3.3. Consider the set of primes

$$
\begin{aligned}
\mathscr{A}= & \left\{Q \in \operatorname{Spec}(R) \mid I_{Q} \text { is not a complete intersection }\right\} \\
& \cap\{Q \in \operatorname{Spec}(R) \mid Q \supset I \text { and } \operatorname{ht}(Q / I)=2\} .
\end{aligned}
$$

By [Sw, Remark (2)] (for example) it follows that $\left\{Q \in \operatorname{Spec}(R) \mid I_{Q}\right.$ is not a complete intersection $\}$ is a Zariski-closed set. Furthermore since $I_{Q}$ is a complete intersection if $\mathrm{ht}(Q / I) \leq 1$ we have that the minimal elements of this Zariski-closed set satisfy $\mathrm{ht}(Q / I) \geq 2$. Thus $\mathscr{A}$ is a finite set (being contained in the family of minimal elements of a Zariski-closed set). As $\mathrm{ht}(M / I)>2$ we have that $M \notin \mathscr{A}$. Furthermore $\operatorname{Ass}\left(R /\left(\left(a_{1}, a_{2}\right)+I^{n}\right)\right) \subseteq X^{4}$ (where 
$\left.X^{4}=\{Q \in \operatorname{Spec}(R) \mid \operatorname{ht}(Q) \leq 4\}\right)$ for each $n \geq 1$ by Proposition 3.5. Therefore by Lemma 2.8 we can choose an element $x \in M \backslash \bigcup_{Q \in \mathscr{A}} Q$ such that $x^{\prime}$ is a $\operatorname{gr}(I, R)$-regular element. Set $I_{1}=(I, x) /(x)$ and $R_{1}=R /(x)$. We claim that $I_{1}$ and $R_{1}$ satisfy the hypotheses of the theorem. If $Q_{1} \supset I_{1}$ and $\operatorname{ht}\left(Q_{1} / I_{1}\right)=$ 1 , then $Q_{1}=Q /(x)$ for some prime ideal $Q$ of $R$ satisfying $\operatorname{ht}(Q / I)=$ 2 (we are using here that $x \notin \bigcup \min (R / I)$ ). Since $x \in Q$ we must have $Q \notin \mathscr{A}$ by our choice of $x$, thus $I_{Q}$ is a complete intersection. It follows that $\left(I_{1}\right)_{Q_{1}}$ is a complete intersection if $\operatorname{ht}\left(Q_{1} / I_{1}\right)=1$. As $x$ is regular on $R / I$ we have that $R_{1} / I_{1} \cong R /(I, x)$ is C-M. From $\mathrm{ht}(I, x)=\mathrm{ht}(I)+1$ it follows that $\mathrm{ht}\left(I_{1}\right)=\mathrm{ht}(I)$ and by Lemma 2.12(ii) we find that $l(I)=l\left(I_{1}\right)$. Finally from the equality $l(I)=l\left(I_{1}\right)$ it follows that $J_{1}=(J, x) /(x)$ is a minimal reduction of $I_{1}$ (because it is generated by $l\left(I_{1}\right)$ elements), and this implies that $r\left(I_{1}\right) \leq 1$. Thus the hypotheses of Theorem 4.1 are satisfied by $I_{1}$ and $R_{1}$. Furthermore $\operatorname{dim}\left(R_{1} / I_{1}\right)=\operatorname{dim}(R / I)-1$, therefore the inductive assumption implies that $R_{1}\left[I_{1} t\right]$ is C-M. It follows that $R[I t]$ is C-M because $R_{1}\left[I_{1} t\right] \cong R[I t] /(x)$ by using that $x^{\prime}$ is a $\operatorname{gr}(I, R)$-regular element. This proves the theorem if $\mathrm{ht}(I)=2$.

Let $\operatorname{ht}(I)=s>2$ and assume the theorem is true for ideals having height at most $s-1$. Let $J$ be a minimal reduction of $I$ satisfying $J I=I^{2}$, and assume $J=\left(a_{1}, \ldots, a_{s}, c, d\right)$ where $\left\{a_{1}, \ldots, a_{s}, c, d\right\}$ is chosen so that it satisfies the conclusions of Proposition 3.3. By Proposition 3.3(viii) we have that $\left(a_{1}, \ldots, a_{s}\right) \cap I^{n}=\left(a_{1}, \ldots, a_{s}\right) I^{n-1}$, thus $\left\{a_{1}^{\prime}, \ldots, a_{s}^{\prime}\right\}$ is a $\operatorname{gr}(I, R)$-regular sequence by Remark 1.11. In particular, $a_{1}^{\prime}$ is a $\operatorname{gr}(I, R)$-regular element. Furthermore, $a_{1}$ is basic at $\mathscr{P}=\{Q \in \operatorname{Spec}(R) \mid Q \supseteq I$ and $\operatorname{ht}(Q / I) \leq 1\}$ by Proposition 3.3(i). Set $R_{2}=R /\left(a_{1}\right)$ and $I_{2}=I /\left(a_{1}\right)$. Then $\left(I_{2}\right)_{Q}$ is a complete intersection for all primes $Q \supset I_{2}$ such that $\mathrm{ht}\left(Q / I_{2}\right)=1$. By using Lemma 2.12(i) and that $a_{1}^{\prime}$ is a $\operatorname{gr}(I, R)$-regular element it follows that $l\left(I_{2}\right) \geq l(I)-1$. But $a_{1}$ is a minimal generator of $J$ and $J_{2}=J /\left(a_{1}\right)$ is a reduction of $I_{2}$, thus $l\left(I_{2}\right)=l(I)-1$ and $J_{2}$ is in fact a minimal reduction of $I_{2}$ (because $J_{2}$ is generated by $l(I)-1$ elements). This shows that $\operatorname{ad}\left(I_{2}\right)=2$. Clearly $J_{2} I_{2}=I_{2}^{2}$, hence $r\left(I_{2}\right) \leq 1$. Therefore the inductive hypotheses (for induction on $h t(I))$ are satisfied for $I_{2}$ and $R_{2}$ so we obtain that $R_{2}\left[I_{2} t\right]$ is C-M. It now follows from Lemma 2.11 that $R[I t]$ is C-M. This completes the proof of Theorem 4.1.

We present some corollaries to Theorem 4.1. These are analogous to the corollaries stated in $\S 2$.

Corollary 4.20. Let $(R, M)$ be a Gorenstein local ring and $P$ a prime ideal of $R$ such that $\operatorname{ad}(P)=2$ and $\operatorname{ht}(P) \geq 2$. Assume that $R / P$ is $C-M$, and $P_{Q}$ is a complete intersection for all prime ideals $Q \supseteq P$ such that $\mathrm{ht}(Q / P)=2$ (e.g., if $R / P$ is nonsingular in codimension 2). Then $R[P t]$ is $C-M$.

Proof. We may pass to $R(X)$ and use Lemma 2.13 to assume that $R / M$ is infinite. By Theorem 1.5(ii) we have that $r(P) \leq 1$, therefore the result follows from Theorem 4.1.

Corollary 4.21. Let $R$ be a Gorenstein local ring and $P$ a prime ideal having deviation at most 2 and height at least 2. Assume that $R / P$ is $C-M$ and $P_{Q}$ is a complete intersection for every prime ideal $Q \supset P$ with $\mathrm{ht}(Q / P)=1$. Then $R[P t]$ is $C-M$. 
Proof. By passing to $R(X)$ and using Lemma 2.13 we may assume that $R / M$ is infinite. If the deviation of $P$ is zero, then $P$ is a complete intersection by Theorem 1.3, therefore $R[P t]$ is C-M by [Ba]. If the deviation of $P$ is 1 , then $P$ has analytic deviation 1 , therefore $R[P t]$ is C-M by Corollary 2.21 . If the deviation of $P$ is 2 , then the analytic deviation of $P$ is either 1 or 2 . If it is 1 , then Corollary 2.21 again shows that $R[P t]$ is $\mathrm{C}-\mathrm{M}$. If it is 2 , then $P$ is its own minimal reduction, therefore $r(P)=0$. In this case $R[P t]$ is $\mathrm{C}-\mathrm{M}$ by Theorem 4.1.

The next corollary shows another instance (along with Corollary 2.23) where the unmixedness of the powers of $P$ forces the Rees algebra of $P$ to be C-M.

Corollary 4.22. Let $R$ be a regular local ring and $P$ a prime ideal of $R$. Assume that $\operatorname{dim}(R / P)=3, R / P$ is normal, and $R / P$ is $C$-M. If $P^{n}=P^{(n)}$ for all $n \geq 1$, then $R[P t]$ is $C-M$.

Proof. By passing to $R(X)$ and using Lemma 2.13 we may assume that $R / M$ is infinite. From Burch's inequality [Bu] $P^{n}=P^{(n)}$ for all $n \geq 1$ implies that $\operatorname{ad}(P) \leq 2$. Also, $P_{Q}$ is a complete intersection if $Q \supset P$ and $h t(Q / P)=1$ because $R / P$ is normal. If $\operatorname{ad}(P)=0$, then $P$ is a complete intersection by [CN], hence $R[P t]$ is $\mathrm{C}-\mathrm{M}$ by [Ba]. If $\operatorname{ad}(P)=1$, then $R[P t]$ is $\mathrm{C}-\mathrm{M}$ by Corollary 2.21. Suppose that $\operatorname{ad}(P)=2$. In order to prove that $R[P t]$ is C-M, it suffices by Theorem 4.1 to show that $r(P) \leq 1$. If $Q \supset P$ and ht $(Q / P)=2$, then $\operatorname{ad}\left(P_{Q}\right) \leq 1$ by Burch's inequality (because $P^{n}=P^{(n)}$ for all $n$ ). Therefore it follows from Theorem 1.5(i) that $r\left(P_{Q}\right) \leq 1$ if $Q \supset P$ and $\operatorname{ht}(Q / P)=2$. The assumptions of [HH, Theorem 3.1] are now satisfied for $P$, hence $r(P) \leq 1$. By Theorem 4.1 it follows that $R[P t]$ is C-M.

We close by mentioning a generic example of an analytic deviation 2 ideal to which our results apply. However this is not a new example. It has been extensively studied.

Example 4.23. Let $K$ be a field and let $R=K\left[X_{i j}\right]$ where $1 \leq i \leq 2$ and $1 \leq j \leq 4$. Set $M=$ the ideal generated by the $X_{i j}$ 's, let $X$ be the $2 \times 4$ generic matrix $\left(X_{i j}\right)$, and let $I$ be the ideal of maximal minors of $X$. Then $\operatorname{ht}\left(I_{M}\right)=3$ and $l\left(I_{M}\right)=5$, hence $\operatorname{ad}\left(I_{M}\right)=2$. Further $I_{Q}$ is a complete intersection if $Q$ is a prime ideal and $Q \neq M$. Also $R / I$ is C-M. Therefore $R_{M}\left[P_{M} t\right]$ is C-M by Corollary 4.20 .

Proof. We refer the reader to $\left[\mathrm{BV}\right.$, Chapter 2] for the statements that $\mathrm{ht}\left(I_{M}\right)=$ $3, R / I$ is C-M, and $I_{Q}$ is a complete intersection for all $Q \neq M$ (and also for a wealth of references and other results on determinantal ideals). The statement that $l\left(I_{M}\right)=3$ was proved in [CN, Proposition].

Remark. The C-M property of $R[I t]$ was also established in [EH, Proposition 2.6].

\section{REFERENCES}

[AH] L. Avramov and J. Herzog, The Koszul algebra of a codimension 2 embedding, Math. Z. 175 (1980), 249-260.

[Ba] J. Barshay, Graded algebras of powers of ideals generated by A-sequences, J. Algebra 25 (1973), 90-99. 
[Br1] M. Brodmann, Asymptotic stability of $\operatorname{Ass}\left(M / I^{n} M\right)$, Proc. Amer. Math. Soc. 74 (1979), 16-18.

[Br2] Rees rings and form rings of almost complete intersections, Nagoya Math. J. 88 (1982), $1-16$.

[BSV] P. Brumatti, A. Simis, and W. V. Vasconcelos, Normal Rees algebras, J. Algebra 112 (1988), 26-48.

[BV] W. Bruns and U. Vetter, Determinantal rings, Lecture Notes in Math., vol. 1327, SpringerVerlag, New York and Berlin, 1988.

[Bu] L. Burch, Codimension and analytic spread, Proc. Cambridge Philos. Soc. 72 (1972), 369373.

[CN] R. Cowsik and M. Nori, On the fibres of blowing up, J. Indian Math. Soc. 40 (1976), 217-222.

[DEP] C. DeConcini, D. Eisenbud, and C. Procesi, Hodge algebras, Astérisque 91 (1982).

[EE] D. Eisenbud and E. G. Evans, Generating modules efficiently: Theorems from algebraic K-theory, J. Algebra 27 (1973), 278-305.

[EH] D. Eisenbud and C. Huneke, Cohen-Macaulay Rees algebras and their specialization, J. Algebra 81 (1983), 202-224.

[EG] E. G. Evans and P. Griffith, Syzygies, London Math. Soc. Lecture Note Ser., no. 106, Cambridge Univ. Press, Cambridge and New York, 1985.

[Fl] H. Flenner, Die Sätze von Bertini für lokale Ringe, Math. Ann. 229 (1977), 97-111.

[GS1] S. Goto and Y. Shimoda, On the Rees algebra of Cohen-Macaulay local rings, Lecture Notes in Pure and Appl. Math., vol. 68, Marcel Dekker, New York, 1979, pp. 201-231.

[GS2] _ On the Gorensteiness of Rees and form rings of almost complete intersections, Nagoya Math. J. 92 (1983), 69-88.

[HI] M. Herrmann and S. Ikeda, On the Gorenstein property of Rees algebras, Manuscripta Math. 58 (1987), 471-490.

[HIO] M. Herrmann, S. Ikeda, and U. Orbanz, Equimultiplicity and blowing up, Springer-Verlag, Berlin, Heidelberg, and New York, 1988.

[HO] M. Herrmann and U. Orbanz, On equimultiplicity, Math. Proc. Cambridge Philos. Soc. 91 (1982), 207-213.

[HRZ] M. Herrmann, J. Ribbe, and S. Zarzuela, On Rees and form rings of almost complete intersections, preprint.

[HSV1] J. Herzog, A. Simis, and W. Vasconcelos, Approximation complexes of blowing up rings, J. Algebra 74 (1988), 466-493.

[HSV2] __, Approximation complexes of blowing up rings. II, J. Algebra 82 (1983), 53-83.

[HVV] J. Herzog, W. Vasconcelos, and R. Villareal, Ideals with sliding depth, Nagoya Math. J. 99 (1985), 159-172.

[HRa] M. Hochster and L. J. Ratliff, Jr., Five theorems on Macaulay rings, Pacific J. Math. 44 (1973), 147-172.

[Hucl] S. Huckaba, On complete $d$-sequences and the defining ideals of Rees algebras, Math. Proc. Cambridge Philos. Soc. 106 (1989), 445-458.

[Huc2] __, Analytic spread modulo an element and symbolic Rees algebras, J. Algebra 128 (1990), 306-320.

[HH] S. Huckaba and C. Huneke, Powers of ideals having small analytic deviation, Amer. J. Math. (to appear).

[Hun1] C. Huneke, On the associated graded ring of an ideal, Illinois J. Math. 26 (1982), 121-137.

[Hun2] __ Symbolic powers of prime ideals and special graded algebras, Comm. Algebra 9 (1981), 339-366.

[Hun3] _ The primary components of and integral closures of ideal in 3-dimensional regular local rings, Math. Ann. 275 (1986), 617-635.

[Hun4] __ Strongly Cohen-Macaulay schemes and residual intersections, Trans. Amer. Math. Soc. 277 (1983), 739-763. 
[HS] C. Huneke and J. Sally, Birational extensions in dimension two and integrally closed ideals, J. Algebra 115 (1988), 481-500.

[HU] C. Huneke and B. Ulrich, Residual intersections, J. Reine Angew. Math. 390 (1988), 1-20.

[IT] S. Ikeda and N. V. Trung, When is the Rees algebra Cohen-Macaulay?, Comm. Algebra 17 (1989), 2893-2922.

[KMU] A. Kustin, M. Miller, and B. Ulrich, Generating a residual intersection, preprint.

[LT] J. Lipman and B. Tessier, Pseudo-rational local rings and a theorem of Briancon-Skoda about integral closures of ideals, Michigan Math J. 28 (1981), 97-116.

[MR] J. Matijevic and P. Roberts, A conjecture of Nagata on graded Cohen-Macaulay rings, J. Math. Kyoto Univ. 14 (1974), 125-128.

[Mat] H. Matsumura, Commutative algebra, 2nd ed., Benjamin/Cummings, Reading, Mass., 1980.

[Mc] S. McAdam, Finite coverings by ideals, Ring Theory (Proc. Oklahoma Conf.), Lecture Notes in Pure and Appl. Math., vol. 7, Marcel Dekker, New York, 1974, pp. 163-171.

[MS] M. Morales and A. Simis, Symbolic powers of monomial curves in $\mathbb{P}^{3}$ lying on a quadric, preprint.

[NR] D. G. Northcott and D. Rees, Reductions of ideals in local rings, Math. Proc. Cambridge Philos. Soc. 50 (1954), 145-158.

[PS] C. Peskine and L. Szpiro, Liaisons des variétés algébriques, Invent. Math. 26 (1974), 271302.

[R1] D. Rees, On a problem of Zariski, Illinois J. Math. 2 (1958), 145-149.

[R2] _ Two classical theorems of ideal theory, Math. Proc. Cambridge Philos. Soc. 52 (1956), 155-157.

[R3] _ A note on analytically unramified local rings, J. London Math. Soc. 36 (1961), 24-28.

[Sa] J. Sally, On the associated graded ring of a local Cohen-Macaulay ring, J. Math. Kyoto Univ. 17 (1977), 19-21.

[Sc] P. Schenzel, Examples of Gorenstein domains and symbolic powers of monomial space curves, preprint.

[SV] A Simis and W. V. Vasconcelos, The syzygies of the conormal module, Amer. J. Math. 103 (1981), 203-224.

[Sw] R. Swan, Serre's problem, (Conf. Commutative Algebra, Kingston, 1975), Queen's Papers on Pure and Appl. Math., no. 42, Queen's Univ., Kingston, Ont., 1975.

[VV] P. Valabrega and G. Valla, Form rings and regular sequences, Nagoya Math. J. 72 (1978), 93-101.

[Ve] J. K. Verma, Rees algebras with minimal multiplicity, Comm. Algebra 17 (12) (1989), 2999-3024. 3027

Department of Mathematics, Florida State University, Tallahassee, Florida 32306 -

E-mail address: huckaba@math.fsu.edu

Department of Mathematics, Purdue University, West Lafayette, Indiana 47907

E-mail address: huneke@math.purdue.edu 